\title{
PERPETUANT SYZYGIES
}

\author{
By A. Young and P. W. Wood.
}

[Received and Read May 12th, 1904.]

The methods used in the following discussion are entirely based on the symbolical notation, perpetuants linear in the coefficients of each quantic concerned being alone considered (perpetuant types). The symbolical forms of perpetuant types (which had previously all been identified by other methods) were calculated by Grace." His result- " Any perpetuant linear in the coefficients of each of $\delta$ quantics, denoted by the letters $a_{1}, a_{2}, \ldots, a_{\delta}$, can be expressed linearly in terms of products and of perpetuants $\left(a_{1} a_{2}\right)^{\lambda_{1}}\left(a_{1} a_{3}\right)^{\lambda_{s}} \ldots\left(a_{1} a_{\delta}\right)^{\lambda_{\delta-1}}$, where $\lambda_{1} \geqslant 2^{\delta-2}, \quad \lambda_{2} \geqslant 2^{\delta-3}, \ldots$, $\lambda_{\delta-1} \geqslant 1$, the order of the letters being fixed beforehand"-is fundamental to our present purpose and will for convenience be quoted as "The Perpetuant Type Theorem."

The difficulty of dealing with the actual syzygies is avoided as follows: all possible products of irreducible forms, for a given degree and weight, are arranged in a predetermined sequence, so that each individual product has a definite place in that sequence. Any syzygy may then be regarded as expressing that one of its products, which comes first in the predetermined sequence, in terms of products which come after it. The first product will be called reducible, by virtue of its being linearly expressible in terms of later products.

Thus, instead of actually finding syzygies, we seek to discover what products are reducible and what products are irreducible. Now all products of perpetuant types of degree $\delta$ can be expressed in terms of the forms

$$
\left(a_{1} a_{2}\right)^{\lambda_{1}}\left(a_{1} a_{8}\right)^{\lambda_{9}} \ldots\left(a_{1} a_{\delta}\right)^{\lambda_{\delta-1}}, \ldots ;
$$

where the indices $\lambda$ are only restricted to be positive integers or zeros, the order of the letters being fixed; such forms are all linearly independent, for, if there is a relation between them, there must be a purely algebraic relation between the symbolical products written down, and this is

- Proc. London Math. Soc., Vol. xxxiv, 
ersily* shown to be impossible. The generating function for all linearly independent perpetuant types and products of perpetuant types of degree $\delta$ is therefore $\frac{1}{(1-x)^{\delta-1}}$; it is known that the generating function for actually irreducible forms of degree $\delta$ is $\frac{x^{2^{\delta-1}}-1}{(1-x)^{\delta-1}}$. So the generating function for irreducible products must be

$$
\frac{1-x^{2^{2-1}-1}}{(1-x)^{\delta-1}}
$$

It is our object to identify these irreducible products : when this has been done for any degree, all the independent syzygies of this degree will have been identified, there being one such syzygy for each reducible proluct of irreducible forms. Now there cannot be fewer irreducible forms (types) and products of degree $\delta$ than the number enumerated by the renerating function $\frac{1}{(1-x)^{\delta-1}}$; hence, when we have reduced all forms except this number, we shall have a proof of the irreducibility of the remianing products, as well as a new proof of the irreducibility of the forms alleady classified as perpetuant types.

Our results are only complete as far as degree 8: a large class of products bas been discussed in general, and, though it cannot be asserted that the generating functions obtained for these products are exact, yet the fact that the particular cases of them are exact as far as degree 8 establishes a strong presumption that such is always the case.

The syzygies are all derived from those of degree 4 due to Stroh, $\ddagger$ and the Jacobian identity

$$
(b c)+(c a)+(a b) \equiv 0 .
$$

The syzygies of degree 4 have been completely discussed already\$ by a different method, but we have discussed them here fully in illustration of the general methods employed in the present paper.

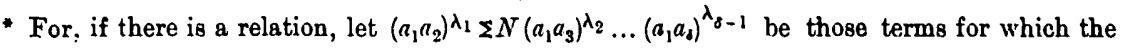
index of $\left(a_{1} a_{2}\right)$ is lowest; then we may divide out by $\left(a_{1} a_{2}\right)^{\lambda_{1}}$ and put $a_{1}=a_{2}$ after division; we get $\Sigma N\left(a_{1} a_{3}\right)^{\lambda_{2}} \ldots\left(a_{1} a_{\delta}\right)^{\lambda_{\delta-1}}=0$. Proceeding in this way, we ultimately see that every coefficient $N$ is zero.

+ The irreducibility of these forms has been finally demonstrated by Wood, "On the Irreducibility of Perpetuant Types," Proc. London Math. Soc., Vol. 1, Ser. 2.

$\ddagger$ Math. Ann., Bd. xxxmr., S. 61-107, § 18.

\$ Wood, Proc. London Math. Sic., Vol, 2, Ser. 2 , 
The above methods are apparently insufficient to obtain all the syzygies for the reduction of products of three forms each of degree 3 , and here arises the difficulty in the treatment of products of degree 9.

It seems probable that the generating function for all irreducible products and types of degree $\delta$, where the products have no factor of degree less than $(n+1)$, is

$$
\frac{x^{\left(\begin{array}{l}
\delta \\
1
\end{array}\right)+\left(\begin{array}{l}
\delta \\
2
\end{array}\right)+. .+\left(\begin{array}{l}
8 \\
x
\end{array}\right)}}{(1-x)^{\delta-1}}, \quad \kappa \leqslant \frac{\delta}{2}
$$

and this result has been proved true for the cases $\kappa=1,2$, and $\kappa \geqslant \frac{\delta}{3}$. The question will be discussed at the end of Section VI.

'The paper has been divided into sections as follows:-

\section{CONTENTS.}

Introduction.

I. Extension of the Perpetuant Type Theorem.

II. Syzygies of Degrees 3 and 4.

III. Definition of Reducibility. General Methods of Reduction by Diffurential Operators.

IV. Syzygies of Degree 5 .

V. (i.) Products having no Factor of Degree less than 2. (ii.) Products haviug no Factor of Degree less than 3.

VI. Syzygies for the Reduction of the Product Forms:-(i.) $C_{\kappa}^{2}$. (ii.) $C_{\alpha} C_{m}\left(\kappa<m<2 \kappa_{\text {; }}\right.$, (iii.) $C_{\mathrm{k}} C_{2 \mathrm{k}}$.

VII. Syzygies of Degrees 6, 7, and 8. Note on Syzygies of Deyree 9.

\section{Previous Literature on Perpetuant. Syzygies.}

Previously published papers, of which none clate since 1887, differ fundamentally from the present in three respects: (1) by the use of the literal notation and the theory of partitions ; (2) by making the discovery of the syzygies a basis for the enumeration of the irreducible perpetuants; (3) by actual expression, apart from mere enumeration, of the syzygies.

The present paper starts from a knowledge of the symbolical form of the perpetuants and deduces a means of enumerating the syzygies; their actual expressions can be obtained from Section III. All the papers quoted are to be found in the American Journal of Mathematics.

Sylvester, Vol. v., "On Subinvariants."

This paper was the starting point of all investigations on perpetuants and treated of certain syzygies of degrees 5,6 , and $7:$ an error in degree 7 was subsequently corrected by Hammond.

Cayley, Vol. vir., "A Memoir on Seminvariants."

This deals with a method of suitably expreseing certain syzygies of degrees $j$ and 6 . 
Hammond, Vol. v., "On the Solution of the Differential Equation of Sources."

This paper obtains the results of Sylvester afresh, and corrects the error in the treatment of degree 7.

_- Vol. vIr., "On Perpetuants, with Applications to the Theory of Finite Quantics."

This paper sketches a general method of classifying and expressing certain syzygies, but gives no definite result for any degree greater than 8 .

MacMahon, Vol. x., "Expression of Syzygies among Perpetuants by means of Partitions."

The paper contains, inter alin, the actual expression of a number of sextic syzygies enumerated by the generating function

$$
\frac{x^{6}+x^{9}+x^{11}+x^{13}+x^{13}+x^{14}+x^{15}+x^{16}+x^{17}}{\left(1-x^{2}\right)\left(1-x^{-4}\right)\left(1-x^{6}\right)}
$$

Several sextic syzygies, however, remain unexpressed.

\section{Extension of the Perpetuant Type Theorem.}

1. Consider any perpetuant

$$
\left(a_{1} a_{2}\right)^{\lambda_{1}}\left(a_{1} a_{3}\right)^{\lambda_{2}} \ldots\left(a_{1} a_{\kappa}\right)^{\lambda_{\kappa-1}} \equiv C_{\kappa} .
$$

We assume throughout that the letters are taken in a definite order fixed beforehand. If $C_{\kappa}$ appears as a factor of a product $P$, we shall find certain conditions, ultimately affecting the indices $\lambda$, for the "irreducibility" of $P$; here we use the word "irreducibility" in a perfectly general sense, as implying that $P$ cannot be expressed in terms of other forms obeying certain definite laws ( $v$. Note, p. 225).

Notation.-The symbol $\left[a_{r_{1}} a_{r_{2}} \ldots a_{r_{1}}\right]$ denotes any covariant, reducible or otherwise, involving the symbolical letters $a_{r_{1}}, a_{r_{2}}, \ldots, a_{r_{l}}$; and the symbol $\left[a_{r_{1}} a_{r_{2}} \ldots a_{r_{1}}\right]^{\prime}$ denotes any covariant, reducible or otherwise, involving all the symbolical letters $a_{1}, a_{2}, \ldots, a_{\kappa}$ concerned except $a_{r_{1}}, a_{r_{2}}, \ldots, a_{r_{l}}$.

If the transvectant $\left(\left[a_{r_{1}} \ldots a_{r_{l}}\right],\left[a_{r_{1}} \ldots a^{r}\right]^{\prime}\right)^{\lambda}$ replace $C_{k}$ in $P$, we may assume from our previous knowledge that, unless $\lambda \geqslant \mu_{r_{1} r_{2} \ldots r_{l}}, P$ is, according to some definition, reducible; here $\mu_{r_{2} r_{2} \ldots r_{l}}$ is a quantity supposed known from other investigations : its value will depend on our definition of "reducibility."

We shall obtain all such transvectants of covariants involving some of the letters of $C_{k}$ with covariants involving the remaining letters, if we suppose that $a_{r_{1}}, a_{r_{3}}, \ldots, a_{r_{l}}$ are some or all of $a_{2}, a_{3}, \ldots, a_{\kappa}$, so that $a_{1}$ appears always in $\left[a_{r_{1}} a_{r_{2}} \ldots a_{r_{1}}\right]^{\prime}$.

To every choice of the letters $a_{r_{1}}, a_{r_{2}}, \ldots, a_{r_{l}}$ corresponds a quantity $\mu_{r_{1} r_{2} \ldots r_{1}}$, so that the number of such quantities $\mu_{r_{1} r_{2} \ldots r_{1}}$ is $2^{k-1}-1$; for 
there are $\left(\begin{array}{c}\kappa-1 \\ l\end{array}\right)$ quantics $\mu_{r_{1}} r_{2} \ldots r_{l}$ with $l$ suffixes, and $l$ may have any value from 1 to $x-1$.

The order of the suffixes may be conveniently fixed by the rule

$$
r_{1}<r_{2}<r_{8} \ldots<r_{l}
$$

We shall use $\sigma_{r}$ to denote the sum of all the $\mu$ 's whose first suffix is $r$, $\sigma_{r, 8}$ to denote the sum of all the $\mu$ 's whose first two suffixes are $r, s$, and so on.

\section{Thus}

$$
\sigma_{k}=\mu_{k}, \quad \sigma_{k-1}=\mu_{k-1}+\mu_{k-1, k},
$$

and so on. We shall establish the following

Theorexr. - The conditions of irreducibility* of the product $P$ are, as far as the factor $C_{k} \equiv\left(a_{1} a_{2}\right)^{\lambda_{1}}\left(a_{1} a_{2}\right)^{\lambda_{2}} \ldots\left(a_{1} a_{k}\right)^{\lambda_{k-1}}$ is concerned,

We have

$$
\lambda_{\kappa-1} \geqslant \sigma_{\kappa}, \quad \lambda_{\kappa-2} \geqslant \sigma_{\kappa-1}, \quad \ldots, \quad \lambda_{1} \geqslant \sigma_{2} .
$$

$$
C_{\kappa} \equiv\left(\left[a_{\kappa}\right]^{\prime},\left[a_{\kappa}\right]\right)^{\lambda_{k-1}}+\Sigma_{\nu}\left(\left[a_{\kappa}\right]^{\prime},\left[a_{k}\right]\right)^{\lambda_{k-1}-\varepsilon},
$$

and therefore by hypothesis $C_{\kappa}$ is reducible unless $\lambda_{k-1} \geqslant \mu_{\kappa}$.

Put

$$
\left(a_{1} a_{2}\right)^{\lambda_{1}}\left(a_{1} a_{3}\right)^{\lambda_{2}} \ldots\left(a_{1} a_{k-2}\right)^{\lambda_{-}}: \equiv \alpha \equiv\left[a_{k-i} a_{k}\right]^{\prime} .
$$

Then $C_{k}$ differs from $\left(a a_{k-1}\right)^{\lambda_{k-2}}\left(u a_{k}\right)^{\lambda_{k-1}}$ by terms which we have just defined as reducible.

If $\lambda_{k-2}+\lambda_{k-1}$ is less than either of $\mu_{k-1}$ or $\mu_{k-1, k}$, then obviously $C_{k}$ is reducible: if $\lambda_{k-2}+\lambda_{k-1}$ is greater than each of $\mu_{k-1}$ and $\mu_{k-1, k}$, then, by Stroh's series, the covariant

$$
\left(\alpha a_{k-1}\right)^{\lambda_{k-1}}\left(\alpha a_{k}\right)^{\lambda_{k-1}}
$$

is linearly expressible in terms of

$$
\begin{aligned}
& \left(a_{k-1} a_{k}\right)^{\lambda_{k}+\lambda_{k-1}},\left(\left(a_{k-1} a_{k}\right)^{\lambda_{k-2}+\lambda_{k-1}-1}, a\right), \ldots \\
& \left(\left(a_{k-1} a_{k}\right)^{\lambda_{k-2}+\lambda_{k-1}-\mu_{k-1, \alpha+1}}, a\right)^{\mu_{k-1, \alpha^{-1}}} \\
& \left(\alpha a_{k}\right)^{\lambda_{k-2}+\lambda_{k-1}},\left(\left(\alpha a_{k}\right)^{\lambda_{k-2}+\lambda_{k-1}-1},\left[a_{k-1}\right]\right), \ldots,\left(\left(\alpha a_{k}\right)^{\lambda_{k-1}+\lambda_{k-1}-\mu_{k-1}+1},\left[a_{k-1}\right]\right)^{\mu_{k-1}-1} ;
\end{aligned}
$$

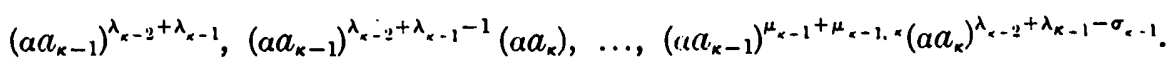

Now by hypothesis each form in the first two rows is reducible, and therefore $\left(\alpha a_{k-1}\right)^{\lambda_{k-2}}\left(\alpha a_{k}\right)^{\lambda_{k-1}}$ is reducible unless $\lambda_{k-2} \geqslant \mu_{k-1}+\mu_{k-1, k}$.

- We always consider $\left(a_{1} a_{2}\right)^{\lambda_{1}}\left(a_{1} a_{3}\right)^{\lambda_{2}} \ldots\left(a_{1} a_{k}\right)^{\lambda_{\alpha}}$, reducible if we can express it in terms of for'ms $\left(a_{1} a_{2}\right)^{\mu_{1}}\left(a_{1} a_{3}\right)^{\mu_{2}} \ldots\left(a_{1} a_{k}\right)^{\mu_{\alpha-1}}$ such that the first of the quantities $\mu_{1}-\lambda_{1}, \mu_{2}-\lambda_{3}, \ldots$, $\mu_{x-1}-\lambda_{x-1}$ which is not zero is positive.

SBR. 2. VOL. 2. NO. 865 . 
Hence a necessary condition for irreducibility is

$$
\lambda_{x-2} \geqslant \sigma_{\kappa-1} \text {. }
$$

Assume the theorem true for the indices $\lambda_{r-1}$ where $r>s$; then, as before, if we put $a \equiv\left[a_{1} a_{2} \ldots a_{s-1}\right]$, we have to consider the form

$$
\left(\alpha a_{s}\right)^{\lambda_{s-1}}\left(\alpha a_{s+1}\right)^{\lambda_{x}} \ldots\left(\alpha a_{x}\right)^{\lambda_{x-1}} \text {. }
$$

By Stroh's series this can be expressed in terms of

1. $\left(a_{s} a_{s+1}\right)^{\lambda_{s}+\lambda_{s-1}}\left[a_{s} a_{s+1}\right]^{\prime},\left(\left[a_{s} a_{s+1}\right],\left[a_{s} a_{s+1}\right]^{\prime}\right), \ldots,\left(\left[a_{s} a_{s+1}\right],\left[a_{s} a_{s+1}\right]^{\prime}\right)^{\sigma_{s, s+1}-1}$;

2. $\left(u a_{s+1}\right)^{\lambda_{s}+\lambda_{s-1}} \ldots,\left(\left[a_{s}\right]^{\prime},\left[a_{s}\right]\right), \ldots,\left(\left[a_{s}\right]^{\prime},\left[a_{s}\right]\right)^{\sigma_{s}-\sigma_{s, s, 1}-1}$;

3. $\left(a a_{s}\right)^{\lambda_{s}+\lambda_{s-1}} \ldots,\left(a a_{s}\right)^{\lambda_{s}+\lambda_{s-1}-1}\left(\alpha a_{s+1}\right) \ldots, \ldots,\left(\alpha a_{s}\right)^{\sigma_{s}}\left(a a_{s+1}\right)^{\lambda_{s}+\lambda_{s-1}-\sigma_{s}}\left(\alpha a_{s+2}\right)^{\lambda_{s+1}} \ldots$.

We proceed to show that each form in the first two rows is reducible.

(1) Any member of the first row is

$$
\left(\left[a_{s} a_{s+1}\right],\left[a_{s} a_{s+1}\right]^{\prime}\right)^{\nu},
$$

where

$$
\begin{gathered}
{\left[a_{i} a_{s+1}\right] \equiv\left(a_{s} a_{s+1}\right)^{\lambda_{k-1}+\lambda_{s}-\nu} \equiv \beta, \text { say, }} \\
{\left[a_{s} a_{s+1}\right]^{\prime} \equiv\left(\alpha a_{s+2}\right)^{\lambda_{s+1}} \ldots\left(a a_{k}\right)^{\lambda_{k-1}},}
\end{gathered}
$$

and

so that we need only consider the form

$$
(u \beta)^{\nu}\left(\alpha a_{s+2}\right)^{\lambda_{+1}} \ldots\left(u a_{k}\right)^{\lambda_{*} \cdot 1} \text {. }
$$

In addition to the actual reductions implied by hypothesis, we must also consider those reductions which consist in increasing the earlier indices at the expense of the later ones. (This is a common feature of all inductive proofs, since these reduced indices may be ruised to their proper limits by virtue of the inductive proof; $v$. Note, p. 225.)

So, since we have assumed the theorem true for the index $\lambda_{s}$ (of $a$ factor followed by not more than $(\kappa-s-1)$ other factors), we must have $\nu \geqslant \sigma_{s, s+1}$, if the form last written down is irreducible. Hence every form in the first row is reducible.

(2) If we put

$$
\beta \equiv\left(\alpha a_{s+1}\right)^{\lambda_{s}+\lambda_{s-1}-\nu},
$$

$$
\left[a_{s}\right]^{\prime} \equiv\left(\beta a_{s+2}\right)^{\lambda_{s+1}}\left(\beta a_{s+3}\right)^{\lambda_{s+2}} \ldots\left(\beta a_{k}\right)^{\lambda_{k-1}} ;
$$

and we have to consider forms

$$
\left(\beta a_{s}\right)^{\nu}\left(\beta a_{s+2}\right)^{\lambda_{s+1}} \ldots\left(\beta a_{k}\right)^{\lambda_{\alpha-1}} .
$$

The condition for the irreducibility of this form is by hypothesis

$\nu^{\prime} \geqslant \operatorname{sum}$ of $\mu$ 's whose first suffix is $s$, and which do not contain a suffix $s+1$,

i.e., $\quad \nu \geqslant \sigma_{s}-\sigma_{s, s+1}$. 
Hence every form in the second row is reducible: and, since the index of $\left(\alpha a_{s}\right)$ in the last row is in every term $\geqslant \sigma_{s}$, we have as the condition, affecting $\lambda_{s-1}$, of irreducibility,

$$
\lambda_{s-1} \geqslant \sigma_{s} .
$$

We have demonstrated the truth of the conditions for the indices $\lambda_{k-1}$ and $\lambda_{x-2}$, and therefore, by induction, the theorem is universally true.

2. Cor. 1.-The perpetuant type theorem is immediately deducible from the preceding result: for, if $C_{\kappa}$ is the only factor of the product $P$, every quantity $\mu$ is unity, since in this case the transvectants are reducible only if they are of zero order.

Hence

$$
\begin{aligned}
\sigma_{s} & =\text { number of ways of choosing all, none, or any of the suffixes } \\
& s+1, \ldots, \kappa, \\
= & 2^{k-s},
\end{aligned}
$$

and therefore the conditions for the irreducibility of $C_{k}$ are

$$
\lambda_{s-1} \geqslant 2^{\kappa-s}, . \quad s=2, \ldots, \kappa .
$$

Cor. 2.-An especially important case arises when the product $P$ is (for any reason) reducible if $C_{k}$ is a Jacobian : in this case every quantity $\mu$ is 2 , and

and therefore

$$
\sigma_{s}=2^{k-s+1} \text {, }
$$

$$
\lambda_{s-1} \geqslant 2^{\kappa-s+1}, \quad s=2, \ldots, \kappa .
$$

In this case the minimum weight of $C_{\kappa}$ for irreducibility is $2^{\kappa}-2$.

(Reference may be made to a paper by Grace, "Extension of Two Theorems on Covariants": Proc. London Math. Soc., Ser. 2, Vol. 1.)

\section{II: Syzygies of Degrees 3 and 4 Respectively.}

3. The syzygies of degrees 3 and 4 offer no difficulties, and may be actually written down apart from the consideration of their reducing special forms : those of degree 4 have been fully discussed already.* A discussion for degree 4 is here given from a different point of view in order to exhibit in a simple case the methods by which syzygies of higher degree are treated.

Degree 3.-There are no syzygies except for weight unity, for which there is a single syzygy :-

$$
(b c)+(c a)+(a b) \equiv 0
$$

*Wood, “On Perpetuant Syzygies of Degree 4," Proc. London Math. Soc., Ser. 2, Vol. 2. 
The generating function for irreducible forms is $\frac{x^{3}}{(1-x)^{2}}$; and, since there are three linearly independent product forms $(b c)^{\omega},(c a)^{\omega},(a b)^{\omega}$ for all values of $\omega$, except $\omega=1,0$, for which there are only two and one respectively, the generating function for irreducible product forms is

$$
3 x^{2} /(1-x)+2 x+1 \text {. }
$$

Hence the generating function for all forms is

$$
\frac{x^{8}}{(1-x)^{2}}+\frac{3 x^{2}}{1-x}+2 x+1=\frac{1}{(1-x)^{2}} \text {. }
$$

4. Degree 4.-There are five different kinds of type forms and products of forms of degree 4 : they may be conveniently written

$$
C_{4}, C_{3}^{2}, C_{1} C_{8}, C_{1}^{2} C_{2}, C_{1}^{4}
$$

where $C_{r}$ denotes any perpetuant of degree $r, C_{r}^{2}$ denotes the product of two perpetuants of degree $r$, and so on. This notation will be preserved throughout.

A product is in the first place considered reducible if it is expressible in terms of products of other kinds which follow it in the above sequence. For weight $\omega$ the syzygies are nine in number and consist of-

(1) The three syzygies, due to Stroh, of the form

$$
\left.\left.a_{1} a_{2} a_{3} a_{4} i_{\omega} \equiv \sum_{i}\left(a_{1} a_{2}\right)+\left(a_{3} a_{4}\right)\right\}^{\omega}-i\left(a_{1} a_{4}\right)+\left(a_{3} a_{2}\right)\right\}^{\omega}=0 \text {. }
$$

(2) The six syzygies, arising from the Jacobian identity, of the form

$$
\left(a_{1} a_{2}\right)^{\omega-1}\left(a_{3} a_{4}\right)=\left(a_{1} a_{2}\right)^{\omega-1}\left(a_{2} a_{4}\right)-\left(a_{1} a_{2}\right)^{\omega-1}\left(a_{2} a_{3}\right) \text {. }
$$

These last syzygies reduce all products $C_{2}^{2}$, when one of the factors $C_{2}$ is a Jacobian, by expressing each such product in terms of products $C_{1} C_{3}$.

In order to fix what products are reduced by Stroh's syzygies, we arrange the products $C_{: 2}^{2}$ in the following order:-

$$
\left(a_{1} a_{4}\right)^{\lambda}\left(a_{2} a_{3}\right)^{\mu}, \quad\left(a_{1} a_{9}\right)^{\lambda}\left(a_{2} a_{4}\right)^{\mu}, \quad\left(a_{1} a_{2}\right)^{\lambda}\left(a_{9} a_{4}\right)^{\mu},
$$

and define-

(i.) $\left(a_{1} a_{4}\right)^{\lambda}\left(a_{2} a_{3}\right)^{\mu}$ as reducible if it is expressible in terms of products $\left(a_{1} a_{3}\right)^{\lambda}\left(a_{2} a_{4}\right)^{\mu},\left(a_{1} a_{2}\right)^{\lambda}\left(a_{3} a_{4}\right)^{\mu}$ and products $C_{1} C_{3}$, \&c.

(ii.) $\left(a_{1} a_{3}\right)^{\lambda}\left(a_{2} a_{4}\right)^{\mu}$ as reducible if it is expressible in terms of products $\left(a_{1} a_{2}\right)^{\lambda}\left(a_{3} a_{4}\right)^{\mu}$ and products $C_{1} C_{3}$, \&c. 
Finally, we shall arrange the products $\left(a_{1} a_{r}\right)^{\lambda}\left(a_{s} a_{t}\right)^{\mu}$ in ascending values of $\lambda$, and define any such form as reducible if it is expressible in terms of similar forms with greater values of $\lambda$.

The syzygy $\left\{a_{1} a_{2} a_{3} a_{4}\right\}_{\omega} \equiv 0$ will reduce the form $\left(a_{1} a_{4}\right)^{2}\left(a_{2} a_{3}\right)^{\omega-2}$, since $\left(a_{1} a_{4}\right)\left(a_{2} a_{9}\right)^{\omega-1}=\Sigma C_{1} C_{3}$, by the Jacobian identity; the syzygy $\left.\vdots a_{1} a_{2} a_{4} a_{3}\right\rangle_{\omega} \equiv 0$ reduces in the same way the form $\left(a_{1} a_{3}\right)^{2}\left(a_{2} a_{4}\right)^{\omega-2}$, and the syzygy $\left\{a_{1} a_{3} a_{2} a_{4}\right\}_{\omega} \equiv 0$ reduces

$$
\left(\begin{array}{l}
\omega \\
2
\end{array}\right)\left(a_{1} a_{4}\right)^{2}\left(a_{2} a_{8}\right)^{\omega-2}+\left(\begin{array}{c}
\omega \\
3
\end{array}\right)\left(a_{1} a_{4}\right)^{8}\left(a_{2} a_{3}\right)^{\omega-3} .
$$

Moreover, from $\left\{a_{1} a_{2} a_{3} a_{4}\right\}_{\omega} \equiv 0$, we have reduced

$$
\begin{aligned}
& \left(\begin{array}{c}
\omega \\
2
\end{array}\right)\left(a_{1} a_{4}\right)^{2}\left(a_{3} a_{2}\right)^{\omega-2}+\left(\begin{array}{c}
\omega \\
3
\end{array}\right)\left(a_{1} a_{4}\right)^{9}\left(a_{3} a_{2}\right)^{\omega-3}, \\
\text { i.e., } & \left(\begin{array}{c}
\omega \\
2
\end{array}\right)\left(a_{1} a_{4}\right)^{2}\left(a_{2} a_{9}\right)^{\omega-2}-\left(\begin{array}{c}
\omega \\
3
\end{array}\right)\left(a_{1} a_{4}\right)^{8}\left(a_{2} a_{9}\right)^{\omega-3} .
\end{aligned}
$$

Hence both $\left(a_{1} a_{4}\right)^{2}\left(a_{2} a_{3}\right)^{\omega-2}$ and $\left(a_{1} a_{4}\right)^{8}\left(a_{2} a_{9}\right)^{\omega-3}$ are, in accordance with our definition, reducible products.

Hence the irreducible products $C_{2}^{2}$ are

$$
\begin{array}{ll}
\left(a_{1} a_{2}\right)^{\lambda}\left(a_{3} a_{4}\right)^{\mu}, & \lambda \geqslant 2, \quad \mu \geqslant 2 ; \\
\left(a_{1} a_{3}\right)^{\lambda}\left(a_{2} a_{4}\right)^{\mu}, \quad \lambda \geqslant 3, \quad \mu \geqslant 2 ; \\
\left(a_{1} a_{4}\right)^{\lambda}\left(a_{2} a_{9}\right)^{\mu}, \quad \lambda \geqslant 4, \quad \mu \geqslant 2 ;
\end{array}
$$

and so the generating function for all products $C_{2}^{2}$ is

$$
\frac{x^{4}}{(1-x)^{2}}+\frac{x^{5}}{(1-x)^{2}}+\frac{x^{6}}{(1-x)^{2}}=\frac{x^{4}-x^{7}}{(1-x)^{9}} .
$$

Finally, when the weight is unity we have only forms $C_{1}^{2} C_{2}$; by the Jacobian transformation, we can compel any one definite letter to appear in the factor $C_{2}$; so for weight unity there are only three such forms, while for all other weights there are six: the generating function for the products $C_{1}^{2} C_{2}$ is therefore

$$
6 x^{2} /(1-x)+3 x
$$


The results for degree 4 may be summarized thus :-

$\begin{array}{cc}\text { Forms. } & \text { Geuerating Functious. } \\ C_{4} & \frac{x^{7}}{(1-x)^{3}} \\ C_{2}^{2} & \frac{x^{4}-x^{7}}{(1-x)^{3}} \\ C_{1} C_{3} & \frac{4 x^{3}}{(1-x)^{2}} \\ C_{1}^{-2} C_{2} & \frac{6 x^{2}}{1-x}+3 x \\ C_{1}^{4} & 1\end{array}$

Hence the generating function for the total number of forms of degree 4 is

$$
\frac{x^{7}+x^{4}-x^{7}}{(1-x)^{3}}+\frac{4 x^{3}}{(1-x)^{2}}+\frac{6 x^{2}}{1-x}+3 x+1=\frac{1}{(1-x)^{8}} .
$$

III. Definition of Redicibility.-General Nethods of Reduction by Differential Operators.

5. The product forms will be arranged in a definite sequence to be particularized immediately, and a product will be defined us reducible if it is linearly expressible in terms of product forms which follow it in this definite sequence. The sequence of the letters involved in a symbolical expression will be that defined by their suffixes, viz., $a_{1}, a_{2}, \ldots, a_{\delta}$.

The criteria for determining the relative positions of any two products $A$ and $B$ in the sequence will be made to depend successively on :-

(i.) The partial degrees of the factors of each product.

(ii.) The arrangement of the letters among the factors of each product.

(iii.) The weights of the factors of each product.

(iv.) The indices of the symbolical determinants of the factors of each product.

(i.) If

$$
\begin{array}{ll}
A \equiv C_{m_{1}} C_{m_{2}} \ldots C_{u_{k_{k}},} & m_{1} \leqslant m_{2} \leqslant m_{3} \ldots \leqslant m_{\kappa} ; \\
B \equiv C_{n_{1}} C_{n_{2}} \ldots C_{n_{l}}, & n_{1} \leqslant n_{2} \leqslant n_{3} \ldots \leqslant n_{l} ;
\end{array}
$$


then $A$ precedes $B$, if the first of the quantities

$$
m_{1}-n_{1}, m_{2}-n_{2}, \ldots, m_{r}-n_{r}, \ldots,
$$

which is not zero, is positive.

(ii.) If

$$
\begin{aligned}
& A \equiv C_{m_{1}} C_{m_{2}} \ldots C_{n_{k}} ! \\
& B \equiv C_{m_{1}}^{\prime} C_{m_{2}}^{\prime} \ldots C_{m_{*}}^{\prime} \int^{\prime}, m_{1} \leqslant m_{2} \leqslant m_{8} \ldots \leqslant m_{k s},
\end{aligned}
$$

so that the factors of $A$ and $B$ are of the same partial degrees, then the arrangement of the letters is taken into consideration.

Case I. $-m_{1}<m_{2}$.

$$
\text { Suppose } \quad C_{m_{1}} \text { contains the letters } a_{r_{1}}, a_{r_{2}}, \ldots, a_{r_{m_{1}}} \text {, }
$$

and

$$
C_{m_{2}}^{\prime} \text { contains the letters } a_{s_{1}}, a_{s_{:}}, \ldots, a_{s_{m_{1}}} \text {; }
$$

where $r_{1}, r_{2}, \ldots, r_{m_{1}}$, or $s_{1}, s_{2}, \ldots, s_{m_{1}}$ are any $m_{1}$ of the suffixes $1,2,3, \ldots, \dot{\delta}$, such that

and

$$
\begin{gathered}
r_{1}<r_{2}<r_{3} \ldots<r_{m_{1}}, \\
s_{1}<s_{2}<s_{3} \ldots<s_{m_{1}} .
\end{gathered}
$$

Then $A$ precedes $B$, if the first of the quantities

$$
r_{1}-s_{1}, r_{2}-s_{2}, \ldots, r_{m_{1}}-s_{m_{1}}
$$

which is not zero is negative.

This assumes that $C_{m_{1}}$ and $C_{m_{1}}^{\prime}$ do not contain the same set of $m_{1}$ letters.

Case II. - $m=m_{1}=m_{2}=\ldots=m_{\theta}<m_{\theta+1} \leqslant m_{\theta+2} \ldots \leqslant m_{\kappa}$.

In the first place the sequence is to be determined as in Case I. by all the letters occurring in the factors $C_{m_{1}} C_{m_{\mathrm{e}}} \ldots C_{m_{\mathrm{q}}}$.

When the letters in $C_{m_{1}} C_{m_{2}} \ldots C_{m_{y}}$ are the same as those in $C_{m_{1}}^{\prime} C_{m_{2}}^{\prime} \ldots C_{m_{2}}^{\prime}$, it is necessary to distinguish between the factors $C_{m_{1}}, C_{m_{2}}, \ldots, C_{n_{b}}$, in order to determine whether $A$ precedes $B$ or not. Let $a_{\phi}$ be the first of the set of letters which occurs in $C_{m_{1}} C_{n_{2}} \ldots C_{m_{\theta}}$; then we define $C_{m_{1}}$ to be that factor which contains $a_{\phi} ;$ similarly $C_{m_{1}}^{\prime}$ is that factor of the product $C_{m_{1}}^{\prime} C_{m_{2}}^{\prime} \ldots C_{m_{w_{4}}}^{\prime}$ which contains $a_{\phi}$. Let the letters of $C_{m_{1}}, C_{m_{1}}^{\prime}$ be respectively $a_{\phi}, a_{r_{2}}, a_{r_{3}}, \ldots, a_{r_{m}}$ and $a_{\phi}, a_{s_{2}}, a_{s_{3}}, \ldots, a_{s_{m}}$, where

$$
\begin{aligned}
& \phi<r_{2}<r_{8} \ldots<r_{m}, \\
& \phi<s_{2}<s_{9} \ldots<s_{m} .
\end{aligned}
$$


Then $A$ precedes $B$ if the tirst of the differences

$$
r_{2}-s_{2}, r_{3}-s_{3}, \ldots, r_{m}-s_{m}
$$

which is not zero is positive.

(As an example, see the arrangement of the sequence of products $C_{2}^{2}$ at the foot of p. 228.)

If the letters of $C_{m_{1}}$ and $C_{n_{1}}^{\prime}$ are the same, we apply the same test to the products $C_{m_{2}} C_{m_{3}} \ldots C_{m_{\theta}}, C_{m_{2}}^{\prime} C_{m_{3}}^{\prime} \ldots C_{m_{\theta}}^{\prime}$. These two products now contain the same set of letters; we choose $C_{m_{2}}, C_{m_{2}}^{\prime}$ to be the two factors which contain the first letter of this set. Then, unless the letters of $C_{m_{2}}, C_{m_{2}}^{\prime}$ are all the same, the sequence is determined by these letters, in the same way as before. Otherwise we must apply the same test to the products $C_{m_{3}} \ldots C_{m_{\theta}}, C_{m_{3}}^{\prime} \ldots C_{m_{\theta}}^{\prime}$.

Finally, when the letters of the various corresponding factors of $C_{m_{1}} C_{m_{2}} \ldots C_{m_{\theta}}$ and $C_{m_{1}}^{\prime} C_{m_{8}}^{\prime} \ldots C_{m_{\theta}}$ are in every case the same, the tests which have just been laid down must be applied to the products $C_{m_{\theta+1}} \ldots C_{m_{k}}$, $C_{m_{\theta+1}}^{\prime} \ldots C_{m_{k}}^{\prime}$ in order to determine whether $A$ precedes $B$ or not.

(iii.) If $A$ and $B$ each have their factors of the same partial degrees in the same sets of letters, let

$$
A \equiv C_{m_{1}} C_{n_{2}} \ldots C_{m_{\varepsilon}}, \quad B \equiv C_{m_{1}}^{\prime} C_{m_{2}}^{\prime} \ldots C_{m_{\varepsilon},}^{\prime} \quad m_{1} \leqslant m_{2} \ldots \leqslant m_{\kappa},
$$

where, if certain of the $m$ 's are equal, the sequence of the factors $C_{m_{1}}, C_{m_{2}}, \ldots, C_{m_{*}}$ is determined as in (ii.), and further let

$$
C_{m_{1}}, C_{m_{2}}, \ldots, C_{m_{k}} \text { be of total weights } \omega_{1}, \omega_{2}, \ldots, \omega_{k} \text { respectively, }
$$

and $C_{m_{1}}^{\prime}, C_{m_{2}}^{\prime}, \ldots, C_{m_{a}}^{\prime}$ be of total weights $\omega_{1}^{\prime}, \omega_{2}^{\prime}, \ldots, \omega_{k}^{\prime}$ respectively.

Then $A$ precedes $B$ if the first of the quantities

$$
\omega_{1}-\omega_{1}^{\prime}, \omega_{2}-\omega_{2}^{\prime}, \ldots, \omega_{k}-\omega_{\kappa}^{\prime}
$$

which is not zero is negative.

(iv.) Finally, we have to consider the case of products whose factors are of the same weights in the same sets of letters. Suppose

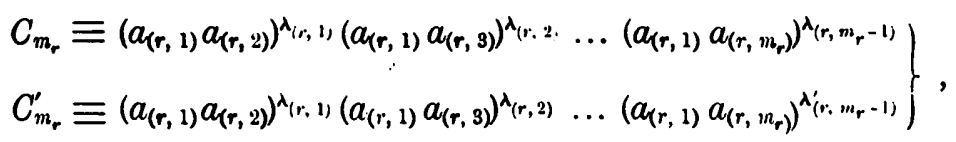

$$
\begin{aligned}
& r=1,2, \ldots, \kappa
\end{aligned}
$$


where $(r, 1)<(r, 2) \ldots<\left(r, m_{r}-1\right)<\left(r, m_{r}\right)$.

Then $A$ precedes $B$, if the first of the quantities

$$
\begin{aligned}
& \text { (1) } \quad \lambda_{(1,1)}-\lambda_{(1,1)}^{\prime}, \quad \lambda_{(1,2)}-\lambda_{(1,2)}^{\prime}, \quad \ldots, \quad \lambda_{\left(1, m_{1}\right)}-\lambda_{\left(1, m_{1}\right)}^{\prime} \text {, } \\
& \text { (2) } \quad \lambda_{(2,1)}-\lambda_{(2,1)}^{\prime}, \quad \lambda_{(2,2)}-\lambda_{(2,2)}^{\prime}, \quad \ldots, \quad \lambda_{\left(2, m_{2}\right)}-\lambda_{\left(2, m_{2}\right)}^{\prime} \text {, } \\
& \text { (r) } \quad \lambda_{(r, 1)}-\lambda_{(r, 1)}^{\prime}, \quad \lambda_{(r, 2)}-\lambda_{(r, 2)}^{\prime}, \quad \ldots, \quad \lambda_{\left(r, m_{r}\right)}-\lambda_{\left(r, m_{r}\right)}^{\prime} \text {, } \\
& \text { (k) } \quad \lambda_{(\kappa, 1)}-\lambda_{(\kappa, 1)}^{\prime}, \quad \lambda_{(\kappa, 2)}-\lambda_{(\kappa, 2)}^{\prime}, \quad \ldots, \quad \lambda_{\left(\kappa, m_{k}\right)}-\lambda_{\left(\kappa, m_{k}\right)}^{\prime}
\end{aligned}
$$

which is not zero is negative.

A reference to the reductions in the case of degree 5 (in the succeeding section) should go some way towards making the above definition of reducibility quite clear.

General Methods of Reduction by the use of Differential Operators.

6. All the reductions made use of depend ultimately on Stroh's syzygies of degree 4 : consider the syzygy

or

$$
\begin{gathered}
\{(a b)+(c d)\}^{\lambda}-\{(a d)+(c b)\}^{\lambda}=0, \\
\sum_{r=0}^{r=\lambda}\left(\begin{array}{l}
\lambda \\
r
\end{array}\right)(a b)^{r}(c d)^{\lambda-r}-\sum_{r=0}^{r=\lambda}\left(\begin{array}{l}
\lambda \\
r
\end{array}\right)(a d)^{r}(c b)^{\lambda-r}=0 .
\end{gathered}
$$

Here the letters $a, b, c, d$ denote any of the letters $a_{1}, a_{2}, \ldots, a_{\delta}$ used elsewhere in the present paper. This syzygy may be written

$$
e^{(a b) D}(c d)^{\wedge}-e^{(n d) B}(c b)^{\wedge}=0,
$$

where

$$
B \equiv x_{1} \frac{\partial}{\partial b_{2}}-x_{2} \frac{\partial}{\partial b_{1}}, \quad D \equiv x_{1} \frac{\partial}{\partial d_{2}}-x_{2} \frac{\partial}{\partial d_{1}} ;
$$

and therefore

$$
B . b_{x}^{m}=0, \quad D(c d)=c_{x}, \quad \ldots
$$

Let $\Gamma_{1}$ be any covariant containing the letters $a, b$, but not the letters $c, d$, and let $\Gamma_{2}$ be any covariant containing the letters $c, d$, but none of the letters of $\Gamma_{1}$. Then we may write

$$
\Gamma_{1}=\Sigma P_{1}(a b)^{\mu_{1}}, \quad \Gamma_{2}=\Sigma P_{2}(c d)^{\mu_{2}},
$$

where $P_{1}$ does not contain the letter $b$ in any determinantal factor, and $P_{2}$ does not contain the letter $d$ in any determinantal factor. 
Then we have a syzygy

$$
P_{1} \cdot e^{(a \omega) D}\left\{P_{2}(c d)^{\lambda}\right\}=P_{1} \cdot e^{(\omega a) B}\left\{P_{2}(c b)^{\lambda}\right\} ;
$$

and this syzygy is a relation between products of covariants of the form $\Gamma_{1} \Gamma_{2}$ and the same products having the letters $b$ and $d$ interchanged.

Now we have arranged our products in a fixed sequence, we shall suppose that the products on the right-hand side of the relation (I.) come after those on the left in this fixed sequence. The syzygy may then be conveniently written

$$
P_{1} e^{(a b) D}\left\{P_{2}(c d)^{\lambda}\right\}=R,
$$

where $R$ denotes any products which come in the fixed sequence after the products on the left-hand side: the sum of the products on the lefthand side is, in fact, according to our definition, reducible.

7. In the first place we shall consider the reduction of products $C_{2} C_{\kappa}$ : let $C_{2} C_{k}=(a b)^{\mu}\left(d_{k} d_{1}\right)^{\lambda_{1}}\left(d_{k} d_{2}\right)^{\lambda_{2}} \ldots\left(d_{k} d_{k-1}\right)^{\lambda_{k-1}}$ : the sequence of such products is primarily determined by the letters contained in the factor $C_{2}$ : we suppose that the product in which the letters are $a b$ precedes any of the products in which the letters of $C_{2}$ are

$$
a d_{1}, a d_{2}, \ldots, a d_{r} \text { respectively. }
$$

This is in accordance with our den̂ned sequence of products when all the letter's $d_{1}, d_{2}, \ldots, d_{r}$ come after the letter $b$. In this case we have a series of relations of the form

$$
e^{(a b) D_{1}} C_{\kappa}=R, \quad e^{(a b) D_{2}} C_{\kappa}=R, \quad \ldots, \quad e^{(a b) D_{r}} C_{\kappa}=R,
$$

where

$$
D_{r}=x_{1} \frac{\partial}{\partial d_{r, 2}}-x_{2} \frac{\partial}{\partial d_{r, 1}} \text {. }
$$

We proceed to show that these $r$ relations are all linearly independent : this result is of fundamental importance.

The operators $D_{1}, D_{2}, \ldots, D_{r}$ are obviously all commutative. The first relation may be written

$$
C_{\kappa}+(a b) \cdot D_{1} C_{\kappa}+\frac{(a b)^{2}}{2 !} D_{1}^{2} C_{\kappa}+\ldots=R,
$$

or, since the first two terms are obviously reducible, the second by the Jacobian identity, it may be written

$$
\frac{(a b)^{2}}{2 !} D_{1}^{2} C_{\kappa}+\frac{(a b)^{3}}{3 !} D_{1}^{3} C_{\kappa}+\ldots=R,
$$

and this relation is true, whatever covariant $C_{x}$ may be involving the letters $d_{1}, d_{2}, \ldots, d_{\kappa}$. 
8. Consider the equation $D_{1} C_{\kappa}=C_{\kappa}^{\prime}$

as an equation to determine $C_{k}$.

If

$$
C_{\kappa}^{\prime}=\left(d_{\kappa} d_{1}\right)^{\mu_{1}}\left(d_{\kappa} d_{2}\right)^{\mu_{3}} \ldots\left(d_{\kappa} d_{\kappa-1}\right)^{\mu_{k-1}},
$$

then one solution is obviously

$$
C_{\kappa}=\frac{1}{\mu_{1}+1}\left(d_{\kappa} d_{1}\right)^{\mu_{1}+1}\left(d_{\kappa} d_{2}\right)^{\mu_{2}} \ldots\left(d_{\kappa} d_{\kappa-1}\right)^{\mu_{\kappa-1}} .
$$

Let $C_{k}^{(1)}$ be any other solution; then we can always write

and hence

$$
C_{\kappa}^{(1)}=\sum_{\rho} N_{\rho}\left(d_{\kappa} d_{1}\right)^{\mu_{\rho, 1}}\left(d_{\kappa} d_{2}\right)^{\mu_{\rho, 2}} \ldots\left(d_{\kappa} d_{\kappa-1}\right)^{\mu_{\rho, \kappa-1}},
$$

$$
\sum_{\rho} N_{\rho} \mu_{\rho, 1}\left(d_{\kappa} d_{1}\right)^{\mu_{\rho, 1}-1}\left(d_{\kappa} d_{2}\right)^{\mu_{\rho, 2}} \ldots\left(d_{\kappa} d_{\kappa-1}\right)^{\mu_{\rho, \alpha-1}} \equiv\left(d_{\kappa} d_{1}\right)^{\mu_{1}} \ldots\left(d_{\kappa} d_{\kappa-1}\right)^{\mu_{\kappa-1}} .
$$

The covariants in this identity are all independent: it follows, therefore, that $C_{\kappa}$ can differ from $\frac{1}{\mu_{1}+1}\left(d_{\kappa} d_{1}\right)^{\mu_{1}+1}\left(d_{\kappa} d_{2}\right)^{\mu_{3}} \ldots\left(d_{\kappa} d_{\kappa-1}\right)^{\mu_{\alpha-1}}$ only by covariants which are products of the quantic $d_{1}$ with covariants of degree $\kappa-1$. And so, neglecting reducible terms, $D_{1}^{-1} C_{\kappa}$ has a unique meaning : in the same way $D_{1}^{-2} C_{\kappa}, D_{1}^{-3} C_{k}, \ldots$ have each a unique meaning. So in (II.) we may replace $C_{\kappa}$ by $D_{1}^{-2} C_{\kappa}$ : the resulting relation is

$$
\frac{(a b)^{2}}{2 !} C_{\kappa}+\frac{(a b)^{3}}{3 !} D_{1} C_{\kappa}+\ldots=R,
$$

and, in the same way, from the relation $e^{(a h) D_{2}} C_{\kappa}=R$ we obtain

$$
\frac{(a b)^{2}}{2 !} C_{\kappa}+\frac{(a b)^{3}}{8 !} D_{2} C_{\kappa}+\ldots=k \text {. }
$$

Therefore, on subtraction,

$$
\frac{(a b)^{3}}{3 !}\left(D_{1}-D_{2}\right) C_{\kappa}+\frac{(a b)^{4}}{4 !}\left(D_{1}^{2}-D_{2}^{2}\right) C_{\kappa}+\ldots=R
$$

or $\left[\frac{(a b)^{3}}{3 !}+\frac{(a b)^{4}}{4 !}\left(D_{1}+D_{2}\right)+\frac{(a b)^{5}}{5 !}\left(D_{1}^{2}+D_{1} D_{2}+D_{2}^{\prime}\right) \ldots\right]\left(D_{1}-D_{2}\right) C_{\kappa}=h$.

Writing this

$$
\chi\left(D_{1}-D_{2}\right) C_{\kappa}=R,
$$

and replacing $C_{\kappa}$ by $D_{1}^{-1} C_{\kappa}$, we have

$$
\chi\left(1-D_{2} D_{1}^{-1}\right) C_{\kappa}=R .
$$

In this replace $C_{\kappa}$ by $D_{2} D_{1}^{-1} C_{\kappa}$ : we have

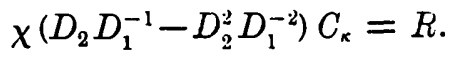


So, adding, we get $\quad \chi\left(1-D_{2}^{2} D_{1}^{-3}\right) C_{\kappa}=R$.

Proceeding in this way, we obtain

$$
\chi\left(1-D_{2}^{\rho} D_{1}^{-\rho}\right) C_{\kappa}=R .
$$

Since $D_{1}^{-\rho}$ has no effect on the index of $\left(d_{k} d_{2}\right)$ in the covariant $C_{k}, \rho$ may be taken so large that $D_{2}^{\rho} D_{1}^{-\rho} C_{\kappa}=0$ : so the last relation is

$$
\chi C_{\kappa}=R,
$$

or

$$
\left[\frac{(a b)^{3}}{3 !}+\frac{(a b)^{4}}{4 !}\left(D_{1}+D_{2}\right)+\ldots\right] C_{\kappa}=R
$$

9. Now let us assume that, in general, we can deduce from the $\sigma$ relations

$$
e^{(a b) D_{1}} C_{\kappa}=R, \quad e^{(a b) D_{2}} C_{\kappa}=R, \quad \ldots, \quad e^{(a l) D_{\sigma}} C_{\kappa}=R
$$

the relation :-

$$
\begin{aligned}
i \frac{(a b)^{\sigma+1}}{(\sigma+1) !}+ & \frac{(a b)^{\sigma+2}}{(\sigma+2) !}\left(D_{1}+D_{2}+\ldots+D_{\sigma}\right)+\ldots \\
& +\frac{(a b)^{\sigma+\tau}}{(\sigma+\tau) !}\left(\sum_{\varpi_{1}+\ldots+\varpi_{\sigma}=\tau-1} D_{1}^{\varpi_{1}} D_{2}^{\sigma_{2}} \ldots D_{\sigma}^{\sigma_{\sigma}}\right)+\ldots j C_{\kappa}=R .
\end{aligned}
$$

We shall show that, if we take another relation $e^{(a b) D_{0+1}} C_{\kappa}=R$, then the resulting relation will be of the same form as (III.) with $\sigma$ changed to $\sigma+1$.

On our assumption we can deduce from the $\sigma$ relations

$$
e^{(n+h) l l_{1}} C_{\kappa}=R, \quad \ldots, \quad e^{(a h) D_{0-1}} C_{\kappa}=R, \quad e^{(a l) D_{\sigma+1}} C_{\kappa}=R
$$

the relation :-

$$
\begin{aligned}
i \frac{(a b)^{\sigma+1}}{(\sigma+1) !} & +\frac{(a b)^{\sigma+2}}{(\sigma+2) !}\left(D_{1}+D_{2}+\ldots+D_{\sigma-1}+D_{\sigma+1}\right)+\ldots \\
& \left.+\frac{(a b)^{\sigma+\tau}}{(\sigma+\tau) !}\left({ }_{\sigma_{1}+\ldots+\varpi_{\sigma-1}+\sigma_{\sigma+1}=\tau-1} D_{1}^{\sigma_{1}} \ldots D_{\sigma-1}^{\varpi_{\sigma-1}} D_{\sigma+1}^{w_{\sigma+1}}\right)+\ldots\right\} C_{\kappa}=R .
\end{aligned}
$$

Subtracting this last relation from (III.), we obtain

$$
\begin{aligned}
& \text { ! } \frac{(a b)^{\sigma+2}}{(\sigma+2) !}+\frac{(a b)^{\sigma+3}}{(\sigma+3) !}\left(\sum_{\rho=1}^{\rho=\sigma+1} D_{\rho}\right)+\ldots \\
& \left.+\frac{(a b)^{\sigma+\tau}}{(\sigma+\tau) !}\left(\sum_{w_{1}+\ldots+w_{\sigma}+w_{\sigma+1}=r-2} D_{1}^{\sigma_{1}} \ldots D_{\sigma+1}^{\sigma_{\sigma+1}}\right)+\ldots\right\}\left(D_{\sigma-1}-D_{\sigma+1}\right) C_{\kappa}=R,
\end{aligned}
$$

and by the same argument as bexiore we can show that $\left(D_{\sigma-1}-D_{\sigma+1}\right) C_{\kappa}$ 
may be replaced by $C_{x}$. Now we have seen that (III.) is true when $\sigma=1,2$ : it is therefore true for all values of $\sigma$.

An immediate consequence of this is the

THEOREM.-The $r$ relations

$$
e^{(a b) D_{1}} C_{\kappa}=R, \quad e^{\left((a l) D_{2}\right.} C_{\kappa}=R, \quad \ldots, \quad e^{(a b) D_{r}} C_{\kappa}=R
$$

are in general linearly independent, and together reduce the product $(a b)^{\lambda} C_{\kappa}$, when $\lambda \leqslant r+1$.

10. Certain special cases remain for consideration.

(i.) The relation $e^{\left(a(l) D_{\alpha}\right.} C_{\kappa}=R$ is true if the product $C_{2} C_{\kappa}$, in which the letters of $C_{2}$ are $(a b)$, precedes the product $C_{2} C_{\kappa}$, in which the letters of $C_{2}$ are $\left(a d_{\kappa}\right)$. We have previously written $C_{\kappa}$ in the form

$$
\left(d_{x} d_{1}\right)^{\lambda_{1}}\left(d_{\kappa} d_{2}\right)^{\lambda_{2}} \ldots\left(d_{k} d_{k-1}\right)^{\lambda_{k}-1}
$$

but it may be equally well written in the form

$$
\sum N\left(d_{1} d_{\kappa}\right)^{\mu_{1}}\left(d_{1} d_{2}\right)^{\mu_{*}} \ldots\left(d_{1} d_{x-1}\right)^{\mu_{x-1}} .
$$

Now the result of operating on each of the terms of this sum is $R$, and therefore the result of operating on the whole sum, or $C_{\kappa}$, is also $R$ : in other words, our preceding results are not afiected by the form of $C_{\kappa}$.

(ii.) If the product $(a b)^{\lambda} C_{k}$ comes before the product $\left(d_{\sigma} b\right)^{\lambda} C_{\kappa}$, we have in the same way another syzygy

or

$$
\begin{aligned}
e^{(k+k) D_{\bullet}} C_{\kappa} & =R \\
e^{(m b)\left(-D_{\odot}\right)} C_{\kappa} & =R ;
\end{aligned}
$$

all such syzygies are independent of those already considered: we may, in fact, write $D_{\kappa+1} \equiv-D_{\sigma}$, and then the extension of the result embodied in (III.) to the case where the operators are $D_{1}, D_{2}, \ldots, D_{\sigma}, D_{\kappa+1}$ is almost identical with the previous investigation. Hence we deduce that, if $r_{1}$ letters of $C_{\kappa}$ come after $a$, and $r_{2}$ letters of $C_{\kappa}$ come after $b$, then

$$
(a b)^{\lambda} C_{\kappa}=R, \quad \text { if } \lambda \leqslant r_{1}+r_{2}+1 .
$$

(iii.) We may suppose that the letters $a$ and $b$ refer to covariants of the original quantics and not to the quantics themselves, and that one of the letters-say, $d_{1}$-refers to a covariant involving the letters, say, $e_{1}, e_{2}$, $\ldots, e_{l}$. Then, if the product $(a b)^{\lambda} C_{\kappa}$ precedes the product $(a d)^{\lambda} C_{\kappa}^{\prime}$, we have a syzygy

$$
e^{(k i))} \nu_{1} C_{k}=l l
$$


or, from the identity $\left(D_{1}+D_{2}+\ldots+D_{x}\right) C_{\kappa}=0$, which is easily verified,

$$
e^{(!(t))\left(-D_{:}-D_{3}-\ldots-D_{k}\right)} C_{\kappa}=R \text {. }
$$

Let $P$ be the covariant obtained from $C_{\kappa}$ by replacing $d_{1}$ by the letters $e_{1}, e_{2}, \ldots, e_{l}$; then

$$
\left(D_{2}+D_{3}+\ldots+D_{\kappa}+E_{1}+E_{2}+\ldots+E_{l}\right) P=0,
$$

and hence the syzygy may be written

$$
e^{(a l)\left(E_{1}+E_{2}+\ldots+E_{1}\right)} C_{\kappa}=R .
$$

Now $C_{\kappa}$ may be represented as a sum of transvectants of covariants of it definite set of $r$ of its letters with covariants of the remainder, and so we may also obtrin syzygies of the form

$$
e^{(a b)\left(D_{1}+D_{2}+\ldots+D_{r}\right)} C_{\kappa}=R,
$$

where $d_{1}, d_{2}, \ldots, d_{r}$ are any of the letter's of $C_{\kappa}$ (the existence of any such relation will, of course, depend on the sequence of the products). We have to show that all such syzygies are linearly independent. We write $D_{\kappa+1}$ for any such composite operator as $\left(D_{1}+D_{2}+\ldots+D_{r}\right)$, and observe that all operator's, simple or composite, are commutative. The proof of the relation (III.) then proceeds on the same lines as before for both simple and composite operators: only one point arises :- Has the operation

$$
D_{\kappa+1}^{-1} C_{\kappa} \equiv\left(D_{1}+D_{2}+\ldots+D_{r}\right)^{-1} C_{\kappa}
$$

a perfectly definite meaning?

To prove that it has, we write $C_{k}$ in the form $\Sigma N(a, \beta)^{\lambda}$, where $\beta$ is a covariant of the letters $d_{1}, d_{2}, \ldots, d_{r}$, and $a$ is a covariant of the remaining letters: then, in the same way as before, we see that

$$
\left(D_{1}+D_{2}+\ldots+D_{r}\right)^{-1}(\alpha \beta)^{\lambda}
$$

differs from $(\lambda+1)^{-1}(\alpha \beta)^{\lambda+1}$ only by products of covariants of $d_{1}, d_{2}, \ldots$, $d_{r}$ with covariants of the remaining letters, and so the meaning is for our purpose definite and unique.

\section{Syzyares of Degree 5.}

11. We shall treat the syzygies of degree 5 at some length in explanation of the general principles set forth in Section III.

The product forms to be considered are of the following classes :-

$$
C_{2} C_{3}, C_{1} C_{4}, C_{1} C_{2}^{2}, C_{1}^{2} C_{3}, C_{1}^{3} C_{2}, C_{1}^{5}
$$

The generating functions for all the irreducible products, except $C_{2} C_{9}$ and $C_{1}^{3} C_{2}$, are known from the results for degrees 3 and 4 . 


\section{(i.) Generating Function for Products $C_{2} C_{3}$.}

The $C_{2} C_{8}$ products are to be arranged in a sequence to be determined thus :

(a) by virtue of the letters involved in the factor $C_{2}$ : the following is the sequence :-

$$
\begin{aligned}
& \begin{array}{llll}
\text { (i.) } & \text { (ii.) (iii.) } & \text { (iv.) } & \text { (v.) }
\end{array} \\
& \left(a_{1} a_{2}\right)^{r} C_{3}, \quad\left(a_{1} a_{3}\right)^{r} C_{3}, \quad\left(a_{1} a_{4}\right)^{r} C_{3}, \quad\left(a_{1} c_{5}\right)^{r} C_{3}, \quad\left(a_{2} a_{3}\right)^{r} C_{9}, \\
& \begin{array}{llll}
\text { (vi.) (vii.) } & \text { (viii.) } & \text { (ix.) } & \text { (x.) }
\end{array} \\
& \left(a_{2} a_{4}\right)^{x} C_{3}, \quad\left(a_{2} a_{5}\right)^{x} C_{3}, \quad\left(a_{3} a_{4}\right)^{x} C_{3}, \quad\left(a_{3} a_{5}\right)^{x} C_{3}, \quad\left(a_{4} a_{5}\right)^{x} C_{3} .
\end{aligned}
$$

(b) If two products $C_{2} C_{3}$ have the same letters in the $C_{2}$ factor, that product whose $C_{2}^{\prime}$ factor is of smaller weight precedes the other.

Any $C_{3} C_{3}$ product is then defined as reducible, if it is expressible linearly in terms of products of other classes and of $C_{2} C_{3}$ products which follow it in the sequence just retermined.

First consider the products $\left(a_{1} a_{2}\right)^{\prime} C_{3}$ : using the notation of the preceding section, we have six linearly independent relations (\$9)

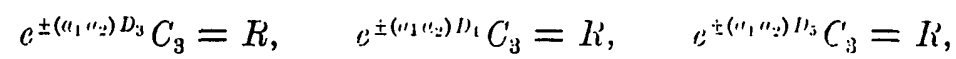

where, for instance, in the relation $e^{\left(\mu_{1} \omega_{:}\right) D_{i n}} C_{3}=R, R$ represents $C_{2} C_{3}$ product forms such as $\left(a_{1} a_{9}\right)^{r} C_{3}$ together with product forms $C_{1} C_{-3}^{\prime \prime} C_{1}^{\prime} C_{3}$, and, in the relation $e^{-\left(\omega_{1} t_{2}\right) D_{:}} C_{3}=R, R$ represents $C_{2} C_{3}$ product forms such as $\left(a_{2}\left(a_{3}\right)^{r} C_{3}\right.$ with product forms $C_{1} C_{2}^{2}, C_{1}^{2} C_{3}$. So, for irreclucibility, we must have $\nu>6+1$, that is, $" \geqslant 8$.

Moreover, $C_{3}$ must not be a Jacobian of the form

$$
\left(\left(a_{r} \cdot a_{\varepsilon}\right)^{\lambda}, a_{t}\right) \text {, where } r, s, t \text { are } 3,4,5 \text { in any order ; }
$$

$$
\text { for } \begin{aligned}
\left(a_{1} a_{2}\right)^{\nu}\left(\left(a_{r} a_{s}\right)^{\lambda}, a^{l}\right) & =\left(a_{r} a_{s}\right)^{\lambda}\left(\left(a_{1} a_{2}\right)^{\prime \prime}, a_{t}\right)-a_{t}\left(\left(a_{1} a_{2}\right)^{\prime \prime},\left(a_{r} \cdot a_{s}\right)^{\lambda}\right) \\
& =R, \quad \text { whatever } r \text { and } s \text { may be. }
\end{aligned}
$$

Hence, by the second Corollary of Section I. (2), the weight of $C_{3}$ is at least 6 , and therefore the minimum weight of an irreducible product $\left(a_{1} a_{2}\right)^{r} C_{3}$ is 14 .

The treatment of the products $\left(a_{1} a_{3}\right)^{\prime} C_{3}$ is similar: here (by $\$$ 9) there are reductions corresponding to the interchange of $a_{3}$ with $a_{4}, a_{5}$, and the interchange of $a_{1}$ with $a_{2}, a_{4}, a_{5}$. The five linearly independent relations are

$$
e^{\left(a_{3}\left(a_{1}\right) D_{2}\right.} C_{3}=R, \quad e^{ \pm\left(a_{1}\left(u_{3}\right) D_{4}\right.} C=R, \quad e^{ \pm\left(u_{1}\left(a_{3}\right) D_{5}\right.} C_{3}=R .
$$

Hence $\nu \geqslant 7$, and, as before, $C_{3}$ must not be a Jacobian of the form $\left(\left(a_{r} a_{s}\right)^{\lambda}, a_{t}\right), r, s, t$ being $2,4,5$ in any order: therefore the minimum 
weight of $C_{3}$ is 6 , and the minimum weight of an irreducible product $\left(a_{1} a_{9}\right)^{\nu} C_{3}$ is 13 .

Similarly for an irreducible product $\left(a_{1} a_{4}\right)^{\nu} C_{3}, \nu \geqslant 6$, and the minimum weight of $C_{3}$ is 6 ; while, for an irreducible product $\left(a_{1} a_{5}\right)^{\nu} C_{3}, \nu \geqslant 5$, and the minimum weight of $C_{3}$ is 6 .

Next consider the products $\left(a_{2} a_{9}\right)^{\nu} C_{3}$ : here there are reductions corresponding to the interchanges of $a_{2}$ with $a_{4}, a_{5}$ and the interchanges of $a_{3}$ with $a_{4}, a_{5}$. The four linearly independent relations are

$$
e^{ \pm\left(a_{*} a_{3}\right) n_{4}} C_{3}=R, \quad e^{ \pm\left(a_{2}\left(r_{3}\right) D_{3}\right.} C_{3}=R ; \quad \text { so that } \nu \geqslant 6 .
$$

Moreover, $C_{3}$ must not be a Jacobian of the form $\left(\left(a_{4} a_{5}\right)^{\lambda}, a_{1}\right)$. Hence, by the second Corollary of Section I. (\$2), the weight of $C_{3}$ is at least $4:$ therefore the minimum weight of an irreducille product $\left(a_{2} a_{3}\right)^{v} C_{3}$ is 10 . In the same way, for an irreducible product $\left(a_{2} a_{4}\right)^{\nu} C_{9}, \nu \geqslant 5$, and the weight of $C_{3} \geqslant 4$, while, for an irreducible product $\left(a_{2} a_{5}\right)^{\nu} C_{3}, \nu \geqslant 4$, and the weight of $C_{3} \geqslant 4$.

Now consider the products $\left(a_{3} a_{4}\right)^{\nu} C_{3}$ : we have for the reduction of such forms two linearly independent relations

$$
e^{ \pm\left(n_{3} n_{4}\right) D_{\mathrm{s}}} C_{3}=R \text {; }
$$

so that $\nu \geqslant 4$, while, by the perpetuant type theorem, $C_{3}$ is of weight 3 at least. So, for $\left(a_{9} a_{5}\right)^{\nu} C_{3}, \nu \geqslant 3$, and $C_{3}$ is of weight 3 at least.

Finally, for the products $\left(a_{4} a_{5}\right)^{\nu} C_{3}$, we have $\nu \geqslant 2$, for, if $\nu=1$, there is a Jacobian identity; also the weight of $C_{3}$ is 3 at least.

12. So the irreducible products $C_{2} C_{8}$ may be tabuiated thus :-

$$
\begin{array}{ll}
\left(a_{1} a_{2}\right)^{\nu}\left(a_{3} a_{4}\right)^{\lambda}\left(a_{3} a_{5}\right)^{\mu}, & \nu \geqslant 8, \quad \lambda \geqslant 4, \quad \mu \geqslant 2 ; \\
\left(a_{1} a_{3}\right)^{\nu}\left(a_{2} a_{4}\right)^{\lambda}\left(a_{2} a_{5}\right)^{\mu}, & \nu \geqslant 7, \quad \lambda \geqslant 4, \quad \mu \geqslant 2 ; \\
\left(a_{1} a_{4}\right)^{\nu}\left(a_{2} a_{9}\right)^{\lambda}\left(a_{2} a_{5}\right)^{\mu}, & \nu \geqslant 6, \quad \lambda \geqslant 4, \quad \mu \geqslant 2 ; \\
\left(a_{1} a_{5}\right)^{\nu}\left(a_{2} a_{3}\right)^{\lambda}\left(a_{2} a_{4}\right)^{\mu}, & \nu \geqslant 5, \quad \lambda \geqslant 4, \quad \mu \geqslant 2 ; \\
\left(a_{2} a_{9}\right)^{\nu}\left(a_{1} a_{4}\right)^{\lambda}\left(a_{1} a_{5}\right)^{\mu}, & \nu \geqslant 6, \quad \lambda \geqslant 3, \quad \mu \geqslant 1 ; \\
\left(a_{2} a_{4}\right)^{\nu}\left(a_{1} a_{3}\right)^{\lambda}\left(a_{1} a_{5}\right)^{\mu}, & \nu \geqslant 5, \quad \lambda \geqslant 3, \quad \mu \geqslant 1 ; \\
\left(a_{2} a_{5}\right)^{\nu}\left(a_{1} a_{3}\right)^{\lambda}\left(a_{1} a_{4}\right)^{\mu}, & \nu \geqslant 4, \quad \lambda \geqslant 3, \quad \mu \geqslant 1 ; \\
\left(a_{3} a_{4}\right)^{\nu}\left(a_{1} a_{2}\right)^{\lambda}\left(a_{1} a_{5}\right)^{\mu}, & \nu \geqslant 4, \quad \lambda \geqslant 2, \quad \mu \geqslant 1 ; \\
\left(a_{3} a_{5}\right)^{\nu}\left(a_{1} a_{2}\right)^{\lambda}\left(a_{1} a_{4}\right)^{\mu}, & \nu \geqslant 3, \quad \lambda \geqslant 2, \quad \mu \geqslant 1 ; \\
\left(a_{4} a_{5}\right)^{\nu}\left(a_{1} a_{2}\right)^{\lambda}\left(a_{1} a_{3}\right)^{\mu}, & \nu \geqslant 2, \quad \lambda \geqslant 2, \quad \mu \geqslant 1 .
\end{array}
$$

Here the minimum value of $\nu$ is determined by the reductions of 
Section III., while the minimum values of the indices $\lambda, \mu$ are determined by the theorem of Section I. ( $§ 1,2)$.

The generating functions for the products $C_{2} C_{3}$ are, therefore, as follows :-

$$
\begin{array}{cccc}
\text { Product. } & \text { G. F. } & \text { Product. } & \text { G. F. } \\
\left(a_{1} a_{2}\right)^{\nu} C_{3} & \frac{x^{14}}{(1-x)^{3}} & \left(a_{2} a_{4}\right)^{\nu} C_{3} & \frac{x^{9}}{(1-x)^{8}} \\
\left(a_{1} a_{8}\right)^{\nu} C_{3} & \frac{x^{13}}{(1-x)^{3}} & \left(a_{2} a_{5}\right)^{\nu} C_{3} & \frac{x^{8}}{(1-x)^{3}} \\
\left(a_{1} a_{4}\right)^{\nu} C_{8} & \frac{x^{12}}{(1-x)^{3}} & \left(a_{3} a_{4}\right)^{\nu} C_{8} & \frac{x^{7}}{(1-x)^{8}} \\
\left(a_{1} a_{5}\right)^{\nu} C_{8} & \frac{x^{11}}{(1-x)^{3}} & \left(a_{3} a_{5}\right)^{\nu} C_{3} & \frac{x^{6}}{(1-x)^{8}} \\
\left(a_{2} a_{8}\right)^{\nu} C_{3} & \frac{x^{10}}{(1-x)^{3}} & \left(a_{4} a_{5}\right)^{\nu} C_{3} & \frac{x^{5}}{(1-x)^{3}}
\end{array}
$$

Hence the generating function for all products $C_{2} C_{3}$ is

$$
\frac{x^{5}+x^{6}+\ldots+x^{19}+x^{14}}{(1-x)^{3}}=\frac{x^{5}-x^{15}}{(1-x)^{4}} .
$$

(ii.) Generating Function for Products $C_{1}^{3} C_{2}$.

Here the only possible reductions arise from the Jacobian identity, when the weight is unity. In this case we can insist on $a_{1}$ appearing in the symbolical determinant factor; so that there are only four linearly independent products of unit weight, while there are ten of any other weight.

Hence the generating function is $10 x^{2} /(1-x)+4 x$.

The generating functions for types and products of degree 5 may be tabulated thus :-

$$
\begin{array}{rlrl}
\text { Forms. } & \text { G. F. } & \text { Forms. } & \text { G. F. } \\
C_{5} & \frac{x^{15}}{(1-x)^{4}} & C_{1}^{2} C_{8} & \frac{10 x^{3}}{(1-x)^{2}} \\
C_{2} C_{8} & \frac{x^{5}-x^{15}}{(1-x)^{4}} & C_{1}^{3} C_{2} & \frac{10 x^{2}}{1-x}+4 x \\
C_{1} C_{4} & \frac{5 x^{7}}{(1-x)^{8}} & C_{1}^{5} & 1 \\
C_{1} C_{2}^{2} & \frac{5\left(x^{4}-x^{7}\right)}{(1-x)^{8}} & &
\end{array}
$$


The sum of all these generating functions is $\frac{1}{(1-x)^{4}}$, which is known to be the generating function for the enumeration of the linearly independent covariants of degree 5 : from this it follows that the perpetuant type of theorem is exact, and that we have found all the syzygies of degree 5 .

V. (i.) Products having no Factor of Degree less than 2.-(ii.) Products having no Factor of Degree less than 3.

13. In the present section we shall show that-

(i.) The generating function for types and irreducible products of degree $\delta$ having no factor $C_{1}$ is

$$
\frac{x^{\delta}}{(1-x)^{\delta-1}}
$$

(ii.) The generating function for types and irreducible products of degree $\delta$ having no factor $C_{1}$ or $C_{2}$ is

$$
\frac{x^{\left(\begin{array}{l}
\delta \\
1
\end{array}\right)+\left(\begin{array}{l}
\delta \\
2
\end{array}\right)}}{(1-x)^{\delta-1}} \text {. }
$$

It seems probable that, in general, the generating function for types and irreducible products of degree $\delta$ having no factor of degree $\kappa$ or less is

$$
\frac{x^{\left(\begin{array}{l}
\delta \\
1
\end{array}\right)+\left(\begin{array}{l}
\delta \\
2
\end{array}\right)+\ldots+\left(\begin{array}{l}
\delta \\
\kappa
\end{array}\right)}}{(1-x)^{\delta-1}}
$$

This general result is verified in Section VI. in the case of

$$
3 \kappa+2 \geqslant \delta \geqslant 2 \kappa \text {. }
$$

(i.) Products having no Factor of Degree less than 2.

14. Those products which do contain a factor $C_{1}$ are all of the form

$$
C_{1}^{r} . P_{\delta-r}, \quad r=1,2, \ldots, \delta-2, \delta .
$$

Let $V_{\delta}$ be the generating function for all types and products not having a factor $C_{1}$; then the expression

$$
V_{\delta}+\left(\begin{array}{l}
\delta \\
1
\end{array}\right) V_{\delta-1}+\left(\begin{array}{l}
\delta \\
2
\end{array}\right) V_{\delta-2}+\ldots+\left(\begin{array}{l}
\delta \\
r
\end{array}\right) V_{\delta-r}+\ldots+\left(\begin{array}{c}
\delta \\
\delta-2
\end{array}\right) V_{2}+V_{0}
$$


is clearly the generating function for all types and irreducible products of degree $\delta$, and so we must have

$$
\frac{1}{(1-x)^{\delta-1}}=V_{\delta}+\left(\begin{array}{l}
\delta \\
1
\end{array}\right) V_{\delta-1}+\ldots+\left(\begin{array}{l}
\delta \\
r
\end{array}\right) V_{\delta-r}+\ldots+\left(\begin{array}{c}
\delta \\
\delta-2
\end{array}\right) V_{2}+V_{0} .
$$

In the first place $V_{0}=1$ : consider $\left(\begin{array}{c}\delta \\ \delta-2\end{array}\right) V_{2}$, the generating function for the products $C_{1}^{\delta-2} C_{2}$; if this product is of unit weight, so that $C_{2}$ is a Jacobian, we can insist on a definite letter being present in the symbolical determinant ; and therefore the coefficient of $x$ in $\left(\begin{array}{c}\delta \\ \delta-2\end{array}\right) V_{2}$ is $(\delta-1)$; for any other weight the products $C_{2} C_{1}^{\delta-2}$ are irreducible, and so

$$
\left(\begin{array}{c}
\delta \\
\delta-2
\end{array}\right) V_{2}=(\delta-1) x+\left(\begin{array}{c}
\delta \\
\delta-2
\end{array}\right) \frac{x^{2}}{1-x} \text {. }
$$

By reference to our previous results, we find that

$$
V_{3}=\frac{x^{8}}{(1-x)^{2}}, \quad V_{4}=\frac{x^{4}}{(1-x)^{3}}, \quad V_{5}=\frac{x^{5}}{(1-x)^{4}} .
$$

Let us assume that

$$
V_{\delta^{\prime}}=\frac{x^{\delta^{\prime}}}{(1-x)^{\delta^{\prime}-1}} \text { for the values } 3,4,5, \ldots, \delta-1 \text { of } \delta^{\prime} \text {; }
$$

then, from the above relation, we have

$$
\begin{aligned}
& V_{\delta}=\frac{\{x+(1-x)\}^{\delta}}{(1-x)^{\delta-1}}-\left\{\left(\begin{array}{l}
\delta \\
1
\end{array}\right) \frac{x^{\delta-1}}{(1-x)^{\delta-2}}+\ldots\right. \\
& +\left(\begin{array}{l}
0 \\
r
\end{array}\right) \frac{x^{\delta-r}}{(1-x)^{\delta-r-1}}+\ldots+(\dot{o}-1) x+1 \\
& =\frac{x^{\delta}}{(1-x)^{\delta-1}} \text {, on expanding the first term. }
\end{aligned}
$$

Honce by induction the result is true in general.

Therefore:-

The generating function for all types and irreducible products of d乞gree $\delta$ which contain no factor of unit degree is $\frac{x^{\delta}}{(1-x)^{\delta-1}}$.

(ii.) Products having no Factor of Degree less than 3.

15. We proceed to find the generating functions for the following products:-
(1) $C_{2}^{m}$,
(2) $C_{2}^{n n} P_{\delta-2 m}$,
(3) $C_{2}^{m} C_{8}$,
(4) $C_{32}^{\text {in }} C_{a}$; 
where $P_{\delta-2 m}$ is any product of degree $\delta-2 m$ which contains no factor of degree $<3$ and is neither $C_{3}$ nor $C_{4}$.

It will be seen that the peculiarity in the treatment of the products $C_{2}^{m} C_{3}$ and $C_{2}^{m} C_{4}$ arises from the Jacobian identity; it may be easily rerified that this special identity yields no exceptional reductions of other products $C_{2}^{m} P_{r}$, even if $P_{r}$ is one of the products $C_{3}^{2}, C_{+}^{2}, C_{3} C_{4}, C_{3} C_{\kappa}$, $C_{4} C_{\kappa}$ (see $\$ 17$, Note).

\section{(1) Generating Function for $C_{2}^{m}$.}

We know that the generating function for $C_{2}^{2}$ is

$$
\frac{x^{4}-x^{7}}{(1-x)^{8}}=\frac{x^{4}(1-x)\left(1-x^{3}\right)}{(1-x)^{4}} .
$$

Consider first the product $C_{2}^{3}$ : we have the following products :-

$$
\left(a_{1} a_{6}\right)^{\lambda} C_{2}^{2}, \quad\left(a_{1} a_{5}\right)^{\lambda} C_{2}^{2}, \quad\left(a_{1} a_{4}\right)^{\lambda} C_{2}^{2}, \quad\left(a_{1} a_{3}\right)^{\lambda} C_{2}^{2}, \quad\left(a_{1} a_{2}\right)^{\lambda} C_{2}^{2} .
$$

Now any of these products is reducible if it is expressible in terms of products following it: thus for $\left(a_{1} a_{2}\right)^{\lambda} C_{2}^{2}$ there is no such reduction, so that $\lambda \geqslant 2$, and the generating function for such products is $\frac{x^{2} V_{2}}{1-x}$, where $V_{2}$ is the generating function of $C_{2}^{2}$; for $\left(a_{1} a_{3}\right)^{\lambda} C_{2}^{2}$ there is a reduction corresponding to the inierchange of $a_{2}$ and $a_{3}$, so that $\lambda \geqslant 3$, and so on ; finally, for $\left(a_{1} a_{6}\right)^{\lambda} C_{2}^{2}$ there are reductions corresponding to the interchanges of $a_{6}$ with $a_{2}, a_{3}, a_{4}, a_{5}$ respectively, and therefore $\lambda \geqslant 6$; hence the generating function for all these products is

$$
\frac{x^{2}+x^{3}+x^{4}+x^{5}+x^{6}}{1-x} V_{2}=\frac{x^{2}-x^{7}}{(1-x)^{2}} V_{2}=\frac{x^{6}(1-x)\left(1-x^{9}\right)\left(1-x^{5}\right)}{(1-x)^{6}}
$$

Assume that the generating function for $C_{2}^{m-1}$ is

$$
V_{m-1}=x^{2 m-2} \frac{(1-x)\left(1-x^{9}\right) \ldots\left(1-x^{2 m-3}\right)}{(1-x)^{2 m-2}} .
$$

Then the products $C_{2}^{\text {in }}$ are

$$
\left(a_{1} a_{2 m}\right)^{\lambda} C_{2}^{m-1}, \quad\left(a_{1} a_{2 m-1}\right)^{\lambda} C_{2}^{m-1}, \ldots, \quad\left(a_{1} a_{r}\right)^{\lambda} C_{2}^{m-1}, \ldots, \quad\left(a_{1} a_{2}\right)^{\lambda} C_{2}^{m-1} .
$$

Here, as before, any product is reducible if it is expressible in terms of products following it: thus for $\left(a_{1} a_{r}\right)^{\lambda} C_{2}^{m-1}$ there are $(r-2)$ reductions corresponding to the interchanges of $a_{r}$ with $a_{2}, a_{3}, \ldots, a_{r-1}$ respectively, and so $\lambda \geqslant r$; for, by Section III., these reductions are all independent; the 
generating function for this set is $\frac{x^{r}}{1-x} V_{m-1}$, and therefore the generating function for all products $C_{\mathbf{2}}^{m}$ is

$$
\begin{aligned}
V_{m} & =\frac{x^{2}+x^{8}+\ldots+x^{2 m}}{1-x} V_{m-1}=\frac{\left(x^{2}-x^{2 m+1}\right)}{(1-x)^{2}} V_{m-1} \\
& =\frac{x^{2 m}(1-x)\left(1-x^{8}\right) \ldots\left(1-x^{2 m-1}\right)}{(1-x)^{2 m}} .
\end{aligned}
$$

Therefore, by induction, the generating function for products $C_{2}^{m}$ is

$$
\frac{x^{2 m}(1-x)\left(1-x^{0}\right) \ldots\left(1-x^{2 m-1}\right)}{(1-x)^{2 m}}
$$

(2) Generating Function for $C_{2}^{m} P_{\delta-2 m}$ (where $P_{\delta-2 m}$ is any form or product, other than $C_{3}$ or $C_{4}$, which contains neither $C_{1}$ nor $C_{2}$ ).

16. Lemma.-If the roots of $y^{n}-p_{1} y^{n-1}+p_{2} y^{n-2}-\ldots+(-)^{n} p_{n}=0$ are $1, x, x^{2}, \ldots, x^{n-1}$, then

$$
p_{\kappa}=x^{\left(\begin{array}{c}
k \\
2
\end{array}\right)} \frac{\left(1-x^{n}\right)\left(1-x^{n-1}\right) \ldots\left(1-x^{n-\kappa+1}\right)}{(1-x)\left(1-x^{2}\right) \ldots\left(1-x^{\kappa}\right)} .
$$

(2) $p_{n}+(1-x) p_{n-2}+(1-x)\left(1-x^{8}\right) p_{n-4}+\ldots$

$$
\begin{aligned}
& +(1-x)\left(1-x^{2}\right) \ldots\left(1-x^{n-1}\right)=1, n \text { even; } \\
& p_{n}+(1-x) p_{n-2}+(1-x)\left(1-x^{9}\right) p_{n-4}+\ldots \\
& +(1-x)\left(1-x^{9}\right) \ldots\left(1-x^{n-2}\right) p_{1}=1, n \text { odd. }
\end{aligned}
$$

(1) Assuming the theorem true for all values up to and including $n$, we shall show that it is also true for the value $(n+1)$; it is obviously true when $n=1,2$.

$$
\begin{aligned}
& \text { If } \begin{aligned}
& y^{n+1}-q_{1} y^{n}+q_{2} y^{n-1}+\ldots+(-)^{n-1} q_{n+1} \\
& \equiv\left(y^{n}-p_{1} y^{n-1}+p_{2} y^{n-2}+\ldots+(-)^{n} p_{n}\right)\left(y-x^{n}\right), \\
& q_{\kappa}=p_{\kappa}+x^{n} p_{\kappa-1}, \quad \kappa=1,2, \ldots, n, \\
& q_{n+1}=p_{n} x^{n} ;
\end{aligned}
\end{aligned}
$$


and therefore, by hypothesis, if $\kappa=1,2, \ldots, n$,

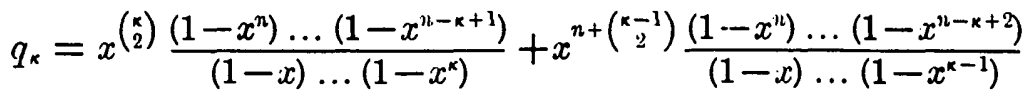

$$
\begin{aligned}
& =x^{(\kappa)} \frac{\left(1-x^{n}\right) \ldots\left(1-x^{n-\kappa+2}\right)}{(1-x) \ldots\left(1-x^{\kappa}\right)}\left\{1-x^{n-\kappa+1}+x^{n-\kappa+1}\left(1-x^{\kappa}\right)\right\} \\
& =x^{\left(\begin{array}{c}
\kappa \\
2
\end{array}\right)} \frac{\left(1-x^{n+1}\right)\left(1-x^{n}\right) \ldots\left(1-x^{n-\kappa+2}\right)}{(1-x)\left(1-x^{2}\right) \ldots\left(1-x^{\kappa}\right)} \text {, }
\end{aligned}
$$

and

$$
q_{n+1}=x^{n} \cdot x^{\left(\begin{array}{l}
n \\
2
\end{array}\right)}=x^{\left(\begin{array}{c}
n+1 \\
2
\end{array}\right)} .
$$

Therefore the result is true universally.

(2) Let the roots of

$$
y^{n+1}-q_{1} y^{n}+\ldots+(-)^{n+1} q_{n+1}=0 \text { be } 1, x, x^{2}, \ldots, x^{n},
$$

and those of

$$
y^{n-1}-r_{1} y^{n-2}+\ldots+(-)^{n-1} r_{n-1}=0 \text { be } 1, x, x^{2}, \ldots, x^{n-2} \text {. }
$$

Assuming the result for the $p$ 's and the $r$ 's, it will be sufficient to prove it for the $q$ 's ; for brevity we consider only the case of $n$ being even. Then

$$
\begin{aligned}
& q_{n+1}+(1-x) q_{n-1}+(1-x)\left(1-x^{3}\right) q_{n-3}+\ldots+(1-x)\left(1-x^{3}\right) \ldots\left(1-x^{n-1}\right) q_{1} \\
& =x^{n} p_{n}+(1-x) \\
& \text { and } \left.\quad p_{n-1}+x^{n} p_{n-2}\right\}+\ldots+(1-x)\left(1-x^{3}\right) \ldots\left(1-x^{n-1}\right)\left(p_{1}+x^{n}\right), \\
& p_{\kappa}=x^{(\kappa)} \frac{\left(1-x^{\prime \prime}\right) \ldots\left(1-x^{n-\kappa+1}\right)}{(1-x) \ldots\left(1-x^{\kappa}\right)}=\frac{1-x^{n}}{1-x^{n-\kappa}} r_{\kappa}
\end{aligned}
$$

by the first part of the Lemma. Therefore

$$
\begin{aligned}
q_{n+1}+ & (1-x) q_{n-1}+\ldots+(1-x)\left(1-x^{3}\right) \ldots\left(1-x^{n-1}\right) q_{1} \\
& =x^{n}\left\{p_{n}+(1-x) p_{n-2}+\ldots+(1-x)\left(1-x^{3}\right) \ldots\left(1-x^{n-1}\right)\right\} \\
& \quad+(1-x) p_{n-1}+(1-x)\left(1-x^{3}\right) p_{n-3}+\ldots+(1-x)\left(1-x^{3}\right) \ldots\left(1-x^{n-1}\right) p_{1} \\
& =x^{n}+\left(1-x^{n}\right)\left\{r_{n-1}+(1-x) r_{n-3}+\ldots+(1-x)\left(1-x^{3}\right) \ldots\left(1-x^{n-3}\right) r_{1}\right\} \\
& =x^{n}+\left(1-x^{n}\right) \text { (by hypothesis) } \\
& =1 .
\end{aligned}
$$

17. Let $V$ be the generating function of $P_{\delta-2 m}, \delta=2 m+r$ being the total degree, and let the letters in $C_{2}^{m}$ be

$$
a_{\kappa_{1}}, a_{\kappa_{2}}, \ldots, a_{\kappa_{2 m}}, \quad \text { where } \kappa_{1}<\kappa_{2}<\kappa_{3} \ldots<\kappa_{2 m} .
$$

Then, by definition, the interchange of the letter $a_{\kappa_{2 m}}$ with any one of the letters $a_{x_{2 n}+1}, a_{x_{3 m}+2}, \ldots, a_{\kappa_{A}}$ implies a reauction, and, by Section III., corre- 
sponding to each of these interchanges the weight of $C_{2}^{m}$ is increased by unity. So in general, by interchanging $a_{x_{2 m} \ldots}$, with succeeding letters occurring in $P_{\delta-2 m}$, the minimum weight for irreducibility is increased by $\left(\delta-\kappa_{2 m-\epsilon}-\epsilon\right)$, since this is the possible number of interchanges. Here $\epsilon$ may be any one of $0,1,2, \ldots, 2 m-1$, and therefore, by interchanging all the letters of $C_{2}^{m}$ with the letters of $P_{\delta-2 m}$ succeeding, the weight is increased by

$$
\left(\delta-\kappa_{1}\right)+\left(\delta-\kappa_{2}\right)+\ldots+\left(\delta-\kappa_{2 m}\right)-\left(\begin{array}{c}
2 m \\
2
\end{array}\right) .
$$

If the $2 m$ letters of $C_{2}^{n 2}$ are fixed, the generating function is

$$
\frac{x^{2 m}(1-x)\left(1-x^{3}\right) \ldots\left(1-x^{2 m-1}\right)}{(1-x)^{2 m-1}} V ;
$$

and therefore, if these $2 m$ letters are chosen in all possible ways and interchanged with the letters of $P_{\delta-2 m}$, the generating function required is

$$
V \cdot x^{-\left(\begin{array}{c}
2 m \\
2
\end{array}\right)} \frac{x^{2 m}(1-x)\left(1-x^{9}\right) \ldots\left(1-x^{2 m-1}\right)}{(1-x)^{2 m-1}} \sum x^{\left(\delta-\kappa_{1}\right)+\left(\delta-\kappa_{2}\right)+\ldots+\left(\delta-\kappa_{2 m}\right)},
$$

where the $\Sigma$ applies to all possible choices of the suffixes $\kappa_{1}, \kappa_{2}, \ldots, \kappa_{2 m}$ from the suffixes $1,2,3, \ldots, \delta$.

The numbers $\delta-\kappa_{1}, \delta-\kappa_{2}, \ldots, \delta-\kappa_{2 m}$ are all different and each is one of the numbers $0,1,2, \ldots, \delta-1$; so, by the Lemma,

$$
\Sigma x^{\delta-\kappa_{1}+\delta-\kappa_{2}+\ldots+\delta-\kappa_{2 m}}=p_{2 m}=x^{\left(\begin{array}{c}
2 m \\
2
\end{array}\right)} \frac{\left(1-x^{\delta}\right) \ldots\left(1-x^{\delta-2 m+1}\right)}{(1-x) \ldots\left(1-x^{2 m}\right)} .
$$

Therefore the generating function for $C_{: 2}^{n} P_{\delta-9 m}$ is

$$
V \frac{x^{2 m}(1-x)\left(1-x^{3}\right) \ldots\left(1-x^{2 m-1}\right)}{(1-x)^{2 ! m}} \frac{\left(1-x^{\delta}\right)\left(1-x^{\delta-1}\right) \ldots\left(1-x^{\delta-2 m+1}\right)}{(1-x)\left(1-x^{2}\right) \ldots\left(1-x^{2 m}\right)},
$$

where $V$ is the generating function for $P_{\delta-2 m}$ and $\delta=2 m+r$ is the total degree.

Note.-If $P_{\delta-2 n}$ contains a factor $C_{3}$ or $C_{4}$ which is a Jacobian, any extra reductions due to a Jacobian transformation will be included in $V$, the generating function for $P_{\delta-2 m}$.

(3) Generating Function for $C_{2}^{m} C_{3}$.

18. A different kind of reduction is introduced only in the case where $C_{3}$ is a Jacooirn : three cases must be separately considered-

(a) $C_{3}$ is a Jacobian and does not contain $a_{1}$ : here there is a new reduction. 
( $\beta$ ) $C_{8}$ is a Jacobian and contains $a_{1}$, but not $a_{2}$ : here there is a new reduction if, and only if, $C_{3}$ is of the form $\left(C_{2}, a_{1}\right)$.

( $\gamma) C_{3}$ contains both $a_{1}$ and $a_{2}$ : here there is never any new reduction.

(a) If the product is irreducible, $C_{8}$ can be written in the form $\left(a_{r_{1}} a_{r_{2}}\right)^{\lambda}\left(a_{r_{1}} a_{r_{3}}\right)^{\mu}, \lambda \geqslant 4, \mu \geqslant 2$, by Section I., Cor. 2.

The number of reductions due to replacing the letters of $C_{3}$ by earlier letters contained in $C_{2}^{m}$ is

$$
\left(r_{1}-1\right)+\left(r_{2}-2\right)+\left(r_{3}-3\right)=\left(r_{1}-2\right)+\left(r_{3}-2\right)+\left(r_{3}-2\right),
$$

and therefore the generating function for such products is

$$
\begin{aligned}
\frac{x^{6}}{(1-x)^{2}} & \frac{x^{2 m}(1-x) \ldots\left(1-x^{2 m-1}\right)}{(1-x)^{2 m}} \sum_{r_{1}, r_{2}, r_{3}=2}^{2 m+3} x^{\left(r_{1}-2\right)+\left(r_{2}-2\right)+\left(r_{3}-2\right)} \\
& =x^{2 m+6} \frac{(1-x)\left(1-x^{3}\right) \ldots\left(1-x^{2 m-1}\right)}{(1-x)^{2 m+2}} \frac{x^{3}\left(1-x^{2 m+2}\right)\left(1-x^{2 m+1}\right)\left(1-x^{2 m}\right)}{(1-x)\left(1-x^{2}\right)\left(1-x^{9}\right)},
\end{aligned}
$$

by the Lemma (\$16).

$(\beta)$ If the product is irreducible, $C_{3}$ can be written in the form

$$
\left(a_{1} a_{r_{1}}\right)^{\lambda}\left(a_{1} a_{r_{2}}\right)^{\mu}, \quad \lambda \geqslant 3, \quad \mu \geqslant 1,
$$

by Section I., $\S 1$, and the number of reductions due to replacing $a_{r_{1}}, a_{r_{2}}$ by earlier letters contained in $C_{2}^{\text {nn }}$ is

$$
\left(r_{1}-2\right)+\left(r_{2}-3\right)=\left(r_{1}-3\right)+\left(r_{2}-3\right)+1 ;
$$

therefore the generating function for such products is

$$
\begin{aligned}
\frac{x^{5}}{(1-x)^{2}} \frac{x^{2 m}(1-x)\left(1-x^{3}\right) \ldots\left(1-x^{2 m-1}\right)}{(1-x)^{2 m}} \sum_{r_{1}, r_{8}=3}^{2 m+3} x^{\left(r_{1}-3\right)+\left(r_{2}-3\right)} \\
=x^{2 m+5} \frac{(1-x)\left(1-x^{3}\right) \ldots\left(1-x^{2 m-1}\right)}{(1-x)^{2 m+2}} \frac{x\left(1-x^{2 m+1}\right)\left(1-x^{2 m}\right)}{(1-x)\left(1-x^{2}\right)} .
\end{aligned}
$$

$(\gamma)$ Here it makes no difference to the irreducibility of the product whether $C_{8}$ is a Jacobian or not; so we may write

$$
C_{8}=\left(a_{1} a_{2}\right)^{\lambda}\left(a_{1} a_{r}\right)^{\mu}, \quad \lambda \geqslant 2, \quad \mu \geqslant 1 ;
$$

and the number of reductions due to replacing $a_{r}$ by earlier letters contained in $C_{2}^{m}$ is $(r-3)$; and therefore the generating function for such products is

$$
\begin{aligned}
\frac{x^{3}}{(1-x)^{2}} \frac{x^{2 m}(1-x)\left(1-x^{9}\right) \ldots\left(1-x^{2 m-1}\right)}{(1-x)^{2 m}} & \sum_{r=3}^{2 m+3} x^{r-3} \\
& =x^{2 m+3} \frac{(1-x)\left(1-x^{8}\right) \ldots\left(1-x^{2 n+1}\right)}{(1-x)^{2 m+8}} .
\end{aligned}
$$


So, finally, the generating function for all products $C_{2}^{m} C_{3}$ is

$$
\begin{aligned}
& \frac{x^{21 x+3}(1-x)\left(1-x^{9}\right) \ldots\left(1-x^{2 n+1}\right)}{(1-x)^{2 m+3}}\left\{1+\frac{x^{3}\left(1-x^{2 n}\right)}{1-x^{2}}+x^{6} \frac{\left(1-x^{2 m}\right)\left(1-x^{2 n+2}\right)}{\left(1-x^{2}\right)\left(1-x^{8}\right)}\right\} \\
& =\frac{x^{\delta}}{(1-x)^{\delta-1}}\left\{(1-x)\left(1-x^{8}\right) \ldots\left(1-x^{\delta-4}\right) p_{3}+(1-x)\left(1-x^{3}\right) \ldots\left(1-x^{\delta-2}\right) p_{1}\right\},
\end{aligned}
$$

where $\delta=2 m+3$ is the total degree, and (as in the Lemma of $\S 16$ )

$$
p_{\kappa}=x^{\left(\begin{array}{c}
\kappa \\
2
\end{array}\right)} \frac{\left(1-x^{\delta}\right)\left(1-x^{\delta-1}\right) \ldots\left(1-x^{\delta-\kappa+1}\right)}{(1-x)\left(1-x^{2}\right) \ldots\left(1-x^{\kappa}\right)} .
$$

(4) Generating Function for $C_{2}^{m} C_{4}$.

19. A different kind of reduction from those considered in $\$ 17$ is introduced when $C_{4}$ is a Jacobian of the form $\left(C_{2}, C_{2}\right)$, and does not contain $a_{1}$, and in no other case.

If $C_{4}$ does not contain $a_{1}$, then, by Section I., if the product is irreducible, it can be written in the form

$$
C_{4} \equiv\left(a_{r_{1}} a_{r_{2}}\right)^{\lambda_{1}}\left(a_{r_{1}} a_{r_{3}}\right)^{\lambda_{2}}\left(a_{r_{1}} a_{r_{1}}\right)^{\lambda_{3}} \text {, where } \lambda_{1} \geqslant 6, \quad \lambda_{2} \geqslant 3, \quad \lambda_{3} \geqslant 1 \text {, }
$$

and, in the same way as before, we find that the generating function for such products is

$$
\begin{aligned}
\frac{x^{10}}{(1-x)^{8}} & \frac{x^{2 m}(1-x)\left(1-x^{9}\right) \ldots\left(1-x^{2 m-1}\right)}{(1-x)^{2 m}} \sum x^{\left(r_{1}-1\right)+\left(r_{2}-2\right)+\left(r_{3}-3\right)+\left(r_{4}-4\right)} \\
& =x^{2 m+10} \frac{(1-x)\left(1-x^{3}\right) \ldots\left(1-x^{2 m-1}\right)}{(1-x)^{2 m+3}} \frac{x^{4}\left(1-x^{2 i n+3}\right) \ldots\left(1-x^{2 i n}\right)}{(1-x)\left(1-x^{2}\right)\left(1-x^{3}\right)\left(1-x^{4}\right)} .
\end{aligned}
$$

If $C_{4}$ does contain the letter $a_{1}$, we write

$$
C_{4} \equiv\left(a_{1} a_{r_{1}}\right)^{\lambda_{1}}\left(a_{1} a_{r_{2}}\right)^{\lambda_{2}}\left(a_{1} a_{r_{3}}\right)^{\lambda_{3}} \text {, where } \lambda_{1} \geqslant 4, \quad \lambda_{2} \geqslant 2, \quad \lambda_{3} \geqslant 1 ;
$$

and the generating function for such products is

$$
\begin{aligned}
\frac{x^{7}}{(1-x)^{8}} & \frac{x^{2 m}(1-x)\left(1-x^{3}\right) \ldots\left(1-x^{2 m-1}\right)}{(1-x)^{2 m}} \Sigma x^{\left(r_{1}-2\right)+\left(r_{2}-3\right)+\left(r_{3}-4\right)} \\
& =x^{2 m+7} \frac{(1-x)\left(1-x^{3}\right) \ldots\left(1-x^{2 m-1}\right)}{(1-x)^{2 m+3}} \frac{\left(1-x^{2 m+9}\right)\left(1-x^{2 m+2}\right)\left(1-x^{2 m+1}\right)}{(1-x)\left(1-x^{2}\right)\left(1-x^{3}\right)} .
\end{aligned}
$$

Hence, adding and simplifying these two results, we find for the complete 
generating function of products $C_{2}^{m} C_{4}$,

$$
\begin{array}{r}
\frac{x^{\delta}}{(1-x)^{\delta-1}}\left\{(1-x)\left(1-x^{3}\right) \ldots\left(1-x^{\delta-5}\right) p_{4}+(1-x)\left(1-x^{3}\right) \ldots\left(1-x^{\delta-3}\right) p_{2}\right. \\
\left.+(1-x)\left(1-x^{3}\right) \ldots\left(1-x^{\delta-1}\right)\left(1-\frac{1}{1-x}\right)\right\},
\end{array}
$$

where $\delta=2 m+4$ is the total degree, and $p_{2}, p_{4}$ are defined above ( $\left.\$ 16\right)$.

20. We are now in a position to find $V_{\delta}$, the generating function for the types and those irreducible products which contain no factor of degree less than 3 ; for we know that $\frac{x^{\delta}}{(1-x)^{\delta-1}}$ is the generating function for the irreducible forms with no factor $C_{1}$, and every such product is of the form $C_{2}^{\text {in }} P_{\delta-2 m}$. We must consider two cases according as $\delta$ is even or odd.

$$
\begin{aligned}
& \quad \delta \text { even. } \\
& \frac{x^{\delta}}{(1-x)^{\delta-1}}=V_{\delta}+\frac{x^{2}(1-x)}{(1-x)^{2}} \frac{\left(1-x^{\delta}\right)\left(1-x^{\delta-1}\right)}{(1-x)\left(1-x^{2}\right)} V_{\delta-2}+\ldots \\
& +\frac{x^{2 m}(1-x)\left(1-x^{3}\right) \ldots\left(1-x^{2 m-1}\right)}{(1-x)^{2 m}} \frac{\left(1-x^{\delta}\right)\left(1-x^{\delta-1}\right) \ldots\left(1-x^{\delta-2 m+1}\right)}{(1-x)\left(1-x^{2}\right) \ldots\left(1-x^{2 m}\right)} V_{\delta-2 m}+\ldots \\
& +\frac{x^{\delta}}{(1-x)^{\delta-1}}\left\{(1-x)\left(1-x^{3}\right) \ldots\left(1-x^{\delta-5}\right) p_{4}+(1-x)\left(1-x^{3}\right) \ldots\left(1-x^{\delta-3}\right) p_{2}\right. \\
& +x^{\delta} \frac{(1-x)\left(1-x^{9}\right) \ldots\left(1-x^{\delta-1}\right)}{(1-x)^{\delta}} ;
\end{aligned}
$$

the last two terms being the generating functions for $C_{2}^{n} C_{4}$ and $C_{2}^{n+2}$ respectively, where $\delta=2 n+4$.

$$
\begin{aligned}
& \quad \text { o odd. }- \\
& \frac{x^{\delta}}{(1-x)^{\delta-1}}=V_{\delta}+\frac{x^{2}(1-x)}{(1-x)^{2}} \frac{\left(1-x^{\delta}\right)\left(1-x^{\delta-1}\right)}{(1-x)\left(1-x^{2}\right)} V_{\delta-2}+\ldots \\
& +\frac{x^{2 m}(1-x)\left(1-x^{3}\right) \ldots\left(1-x^{2 m-1}\right)}{(1-x)^{2 m}} \frac{\left(1-x^{\delta}\right)\left(1-x^{\delta-1}\right) \ldots\left(1-x^{\delta-2 m+1}\right)}{(1-x)\left(1-x^{2}\right) \ldots\left(1-x^{22 n}\right)} V_{\delta-3 m}+\ldots \\
& +\frac{x^{\delta}}{(1-x)^{\delta-1}}(1-x)\left(1-x^{3}\right) \ldots\left(1-x^{\delta-1}\right) p_{3}+(1-x)\left(1-x^{3}\right) \ldots\left(1-x^{\delta-2}\right) p_{1}^{\prime} ;
\end{aligned}
$$

the last term being the generating function for $C_{2}^{n} C_{3}$, where $\delta=2 n+3$. 
Now assume that, for $r<\delta$ and $>4$,

$$
V_{r}=\frac{x^{\left(\begin{array}{c}
r \\
1
\end{array}\right)+\left(\begin{array}{c}
r \\
2
\end{array}\right)}}{(1-x)^{r-1}},
$$

and it is easily verified, from the two results of the preceding Lemma (\$16), that, whether $\delta$ is even or odd,

$$
V_{\delta}=\frac{x^{\left(\begin{array}{l}
(\delta) \\
1
\end{array}\right)+\left(\begin{array}{l}
\delta \\
2
\end{array}\right)}}{(1-x)^{\delta-1}} .
$$

Hence the result is established in general.
VI. Products of the kinds
(1) $C_{\kappa}^{2}$;
(2) $C_{\kappa} C_{m}, \kappa<m<2 \kappa$; (3) $C_{\kappa} C_{2 \kappa}$.

21. The general principle involved by the present application of Stroh's syzygies of degree 4 may be explained thus: Suppose we are seeking for the syzygies in which a product $C_{\kappa} C_{m}$ occurs. Let us divide up the letters of $C_{\kappa}$ into two sets,

$$
a_{1}, a_{2}, \ldots, a_{r_{1}}, \quad b_{1}, b_{2}, \ldots, b_{r_{2}} ;
$$

and those of $C_{m}$ into two sets,

$$
c_{1}, c_{2}, \ldots, c_{s_{1}}, \quad d_{1}, d_{2}, \ldots, d_{s_{2}} .
$$

Let $a, \beta, \gamma, \delta$ be covariants, whose symbolical characters are comprised in the sets $a_{1}, a_{2}, \ldots, a_{r_{1}}: b_{1}, b_{2}, \ldots, b_{r_{2}}: c_{1}, c_{2}, \ldots, c_{s_{1}}: d_{1}, d_{2}, \ldots, d_{s_{2}}$ respectively. Then $C_{k}$ can be expressed in terms of transvectants of the form $(\alpha, \beta)^{\lambda}$, and $C_{m}$ can be expressed in terms of transvectants of the form $(\gamma, \delta)^{\mu}$.

Consider the Stroh syzygy

$$
\{(\alpha \beta)+(\gamma \delta)\}^{\omega}-\{(\alpha \delta)+(\gamma \beta)\}^{\omega}=0,
$$

which may be written $e^{(a \beta) D_{s}}(\gamma \delta)^{\omega}=e^{(a \delta) D_{s}}(\gamma \beta)^{\omega}$,

where

$$
\begin{aligned}
& D_{\beta}=x_{1} \frac{\partial}{\partial \beta_{2}}-x_{2} \frac{\partial}{\partial \beta_{1}}, \\
& D_{\delta}=x_{1} \frac{\partial}{\partial \delta_{2}}-x_{2} \frac{\partial}{\partial \delta_{1}} .
\end{aligned}
$$

Now let us suppose $(\alpha \delta)^{\rho}(y \beta)^{\sigma}$ is a product which, by the definition of Section III., succeeds the product $(\alpha \beta)^{\rho^{\prime}}(\gamma \delta)^{\sigma^{\prime}}$ in the sequence of products : then we have the relation

or

$$
\begin{aligned}
e^{(\alpha \beta) D_{o}}(\gamma \delta)^{\omega} & =\text { reducible terms } \\
e^{(\alpha \beta) D_{o}}\left(c_{1}, c_{2}, \ldots, c_{s_{1}}, \delta^{\prime}\right) & =\text { reducible terms }
\end{aligned}
$$


where $\left(c_{1}, c_{2}, \ldots, c_{s_{1}}, \delta\right)$ is the symbolical product obtained by replacing $\gamma$ by the letters of which it is composed. Moreover, since $c_{1}, c_{2}, \ldots, c_{s_{1}}, \delta$ are the only letters involved in this symbolical product, we know that

$$
\left(D_{c_{1}}+D_{c_{2}}+\ldots+D_{c_{s_{1}}}+D_{\delta}\right)\left(c_{1}, c_{2}, \ldots, c_{s_{1}}, \delta\right)=0 .
$$

Therefore we may write the relation

or

$$
\left.\begin{array}{c}
e^{-(a \beta)\left\{D_{c_{1}}+D_{c_{2}}+\ldots+D_{s_{s_{1}}}\right\}} C_{m}=\text { reducible terms } \\
e^{(a \beta)\left\{D_{d_{d_{1}}}+D_{d_{d_{2}}}+\ldots+D_{d_{t_{2}}}\right\}} C_{m}=\text { reducible terms }
\end{array}\right\} .
$$

This relation gives us a reduction for the product $C_{k} C_{m}$ by virtue of the fact that the product

$$
\left(a_{1}, a_{2}, \ldots, a_{r_{1}}, b_{1}, b_{2}, \ldots, b_{r_{2}}\right)\left(c_{1}, c_{2}, \ldots, c_{s_{1}}, d_{1}, d_{2}, \ldots, d_{s_{2}}\right)
$$

precedes, in our fixed arrangement, the product

$$
\left(a_{1}, a_{2}, \ldots, a_{r_{1}}, d_{1}, d_{2}, \ldots, d_{s_{2}}\right)\left(b_{1}, b_{2}, \ldots, b_{r_{1}}, c_{1}, c_{2}, \ldots, c_{s_{1}}\right) .
$$

In this way we obtain a series of relations such as (1): such relations have been considered in Section III. [ $\S 10$, (iii.)], and have there been shewn to be all independent. It follows, therefore, that we obtain one reduction for $C_{\kappa} C_{m}$ corresponding to every product $C_{\mathrm{k}^{\prime}} C_{m^{\prime}}$ following $C_{\mathrm{k}} C_{m}$ in the sequence of products already determined, provided that each of $C_{\kappa^{\prime}}, C_{m^{\prime}}$ contains letters from each of the factors $C_{k}, C_{m}$; and for every such reduction the minimum weight of $C_{\kappa}$ for irreducibility is increased by unity.

\section{(i.) Generating Function for $C_{\kappa^{*}}^{2}$}

22. Let

$$
\begin{aligned}
C_{\kappa}^{2} & \equiv\left(a_{1} a_{r_{1}}\right)^{\lambda_{1}}\left(a_{1} a_{r_{2}}\right)^{\lambda_{2}} \ldots\left(a_{1} a_{r_{k-1}}\right)^{\lambda_{k-1}}\left(b_{1} b_{2}\right)^{\mu_{1}}\left(b_{1} b_{8}\right)^{\mu_{8}} \ldots\left(b_{1} b_{k}\right)^{\mu_{k-1}}, \\
& \equiv C_{k}^{(1)} C_{\kappa}^{(2)},
\end{aligned}
$$

where $r_{1}<r_{2}<\ldots<r_{\kappa-1}$, and the letters involved are $a_{1}, a_{2}, \ldots, a_{2 k}$ in some order. If either $C_{\kappa}^{(2)}$ or $C_{\kappa}^{(2)}$ is a Jacobian, the product is reducible, since it is expressible in terms of products $C_{x-e} C_{x+e}$ : hence, by the second Corollary of Section I., each of $C_{k}^{(1)}$ or $C_{k}^{(2)}$ is of weight $\left(2^{k}-2\right)$ at least.

In the first place we suppose the letters of $C_{k}^{(1)}$ and of $C_{k}^{(2)}$ to be fixed and we disregard their arrangement: we then find how many reductions are possible by virtue of expressing $C_{k}^{(1)} C_{k}^{(2)}$ in the form $\Sigma C_{k-e} C_{\kappa+e \cdot}$ To this end we consider all possible products $C_{r} C_{2 k-r}$, such that $r$ takes any value from 2 to $\kappa-1$, where each of $C_{r}, C_{2 \kappa-r}$ contains letters from each of the factors $C_{k}^{(1)}, C_{k}^{(2)}$ : then we know that, corresponding to every such product, there is a reduction for $C_{k}^{(1)} C_{k}^{(2)}$, and that these reductions are all 
independent; so that, corresponding to each such product, the minimum weight of $C_{k}^{(1)}$ for the irreducibility of $C_{k}^{(1)} C_{k}^{(2)}$ is increased by unity.

The letters of $C_{r}$ may be chosen in $\left(\begin{array}{c}2 \kappa \\ r\end{array}\right)$ ways, but we must exclude every cuse where these $r$ letters are all included among the letters of $C_{k}^{(1)}$ or among those of $C_{x}^{(2)}$, for in such cases there is no corresponding reduction of the product $C_{\kappa}^{(1)} C_{\kappa}^{(2)}$; hence the number of choices for $C_{r} C_{I_{k}-r}$ is

$$
\left(\begin{array}{c}
2 \kappa \\
r
\end{array}\right)-\left(\begin{array}{l}
\kappa \\
r
\end{array}\right)-\left(\begin{array}{l}
\kappa \\
r
\end{array}\right)
$$

So the total number of reductions of this kind for $C_{k}^{(1)} C_{k}^{(2)}$ is

$$
\begin{aligned}
\sum_{r=2}^{r=\kappa-1}\left\{\left(\begin{array}{c}
2 \kappa \\
r
\end{array}\right)-2\left(\begin{array}{l}
\kappa \\
r
\end{array}\right)\right\} & =\frac{1}{2}\left[2^{2 \kappa}-4 \kappa-2-\left(\begin{array}{c}
2 \kappa \\
\kappa
\end{array}\right)\right]-2\left[2^{\kappa}-\kappa-2\right] \\
& =2^{2 \kappa-1}-2^{\kappa+1}-\frac{1}{2}\left(\begin{array}{c}
2 \kappa \\
\kappa
\end{array}\right)+3 .
\end{aligned}
$$

Further, by the Jacobian identities, which are independent of these reductions, the minimum weight of $C_{x}^{(1)}$ is $2^{x}-2$. So, whatever are the letters of $C_{k}^{(1)}$, the minimum weight of $C_{k}^{(1)}$ to ensure the irreducibility of $C_{k}^{(1)} C_{k}^{(2)}$ is

$$
2^{2 \kappa-1}-2^{\kappa}-\frac{1}{2}\left(\begin{array}{c}
2 \kappa \\
\kappa
\end{array}\right)+1
$$

It remains to discover how many products $P_{1} P_{2}$ follow $C_{k}^{(1)} C_{k}^{(2)}$ in the sequence of products already determined, where $P_{1}$ and $P_{2}$ are each of degree $\kappa$, and $P_{1}$ is taken as that factor which contains $a_{1}$.

If $r_{1}>2$, there is a reduction corresponding to every product $P_{1} P_{2}$, where $P_{1}$ contains $a_{2}$, and the number of such products is $\left(\begin{array}{c}2 \kappa-2 \\ \kappa-2\end{array}\right)$.

If $r_{1}>3$, there is a reduction corresponding to every product $P_{1} P_{2}$, where $P_{1}$ contains $a_{3}$ and not $a_{2}$, and the number of such products is $\left(\begin{array}{c}2 \kappa-3 \\ \kappa-2\end{array}\right)$.

Continuing in this way, we obtain

$$
\left(\begin{array}{c}
2 \kappa-2 \\
\kappa-2
\end{array}\right)+\left(\begin{array}{c}
2 \kappa-3 \\
\kappa-2
\end{array}\right)+\ldots+\left(\begin{array}{c}
2 \kappa-r_{1}+1 \\
\kappa-2
\end{array}\right)=\left(\begin{array}{c}
2 \kappa-1 \\
\kappa-1
\end{array}\right)-\left(\begin{array}{c}
2 \kappa-r_{1}+1 \\
\kappa-1
\end{array}\right)
$$

reductions.

If we have any further reductions, $P_{1}$ must contain both $a_{1}$ and $a_{r_{1}}$, and we get a reduction if $P_{1}$ contains also $a_{i z+1}$ where $r_{1}+1<r_{z}$; in 
this way we have

$$
\left(\begin{array}{c}
2 \kappa-r_{1}-1 \\
\kappa-3
\end{array}\right)+\left(\begin{array}{c}
2 \kappa-r_{1}-2 \\
\kappa-3
\end{array}\right)+\ldots+\left(\begin{array}{c}
2 \kappa-r_{2}+1 \\
\kappa-3
\end{array}\right)=\left(\begin{array}{c}
2 \kappa-r_{1} \\
\kappa-2
\end{array}\right)-\left(\begin{array}{c}
2 \kappa-r_{2}+1 \\
\kappa-2
\end{array}\right)
$$

reductions.

The same argument is pursued for each of the letters of $C_{k}^{(1)}$ in turn: hence the number of reductions, corresponding to the products $C_{\kappa}^{2}=P_{1} P_{2}$, which follow $C_{k}^{(1)} C_{k}^{(2)}$ in the determined sequence is

$$
\begin{array}{r}
\left(\begin{array}{c}
2 \kappa-1 \\
\kappa-1
\end{array}\right)-\left(\begin{array}{c}
2 \kappa-r_{1}+1 \\
\kappa-1
\end{array}\right)+\left(\begin{array}{c}
2 \kappa-r_{1} \\
\kappa-2
\end{array}\right)-\left(\begin{array}{c}
2 \kappa-r_{2}+1 \\
\kappa-2
\end{array}\right)+\ldots-\left(\begin{array}{c}
2 \kappa-r_{\kappa-1}+1 \\
1
\end{array}\right) \\
=\left(\begin{array}{c}
2 \kappa-1 \\
\kappa-1
\end{array}\right)-\left(\begin{array}{c}
2 \kappa-r_{1} \\
\kappa-1
\end{array}\right)-\left(\begin{array}{c}
2 \kappa-r_{2} \\
\kappa-2
\end{array}\right)-\left(\begin{array}{c}
2 \kappa-r_{3} \\
\kappa-3
\end{array}\right)-\ldots-\left(\begin{array}{c}
2 \kappa-r_{\kappa-1} \\
1
\end{array}\right)-1 .
\end{array}
$$

Hence, if the letters of $C_{\kappa}^{(1)}$ are $a_{1}, a_{r_{1}}, \ldots, a_{r_{k-1}}$, its minimum weight is

$$
2^{2 \kappa-1}-2^{\kappa}-\left[\left(\begin{array}{c}
2 \kappa-r_{1} \\
\kappa-1
\end{array}\right)+\left(\begin{array}{c}
2 \kappa-r_{2} \\
\kappa-2
\end{array}\right)+\ldots+\left(\begin{array}{c}
2 \kappa-r_{k-1} \\
1
\end{array}\right)\right],
$$

and, since the minimum weight of $C_{x}^{(2)}$ is $\left(2^{\kappa}-2\right)$, the generating function for the irreducible products $C_{k}^{2}$ is

$$
\frac{x^{2^{2 \alpha-1}-2}}{(1-x)^{2 x-2}} \sum_{r_{1}, r_{2}, \ldots, r_{\kappa-1}} x^{-\left(\begin{array}{c}
2 x-r_{1} \\
x-1
\end{array}\right)-\left(\begin{array}{c}
2 x-r_{2} \\
x-2
\end{array}\right)-\ldots-\left(\begin{array}{c}
2 x-r_{\alpha-1} \\
1
\end{array}\right)}
$$

where $r_{1}, r_{2}, \ldots, r_{k-1}$ take all values satisfying

$$
1<r_{1}<r_{2}<\ldots<r_{k-1}<2 \kappa+1 .
$$

We proceed to show that

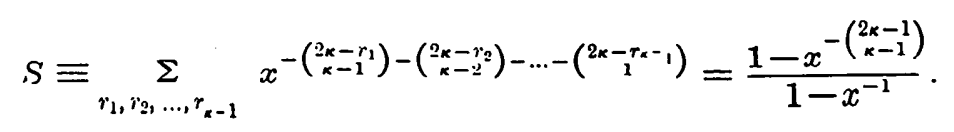

First find the sum for the possible values $r_{k-2}+1, r_{k-2}+2, \ldots, 2 \kappa$ of $r_{k-1}$; we have

and therefore

$$
\sum_{r_{*-1}} x^{-\left(2 \kappa-r_{x-1}\right)}=\frac{1-x^{-\left(2 k-r_{\alpha-2}\right)}}{1-x^{-1}} ;
$$

$$
\begin{aligned}
& S \equiv \frac{1}{1-x^{-1}}\left\{\sum _ { r _ { 1 } , r _ { 2 } , \ldots , r _ { k - 2 } } \left(x^{-\left(\begin{array}{c}
2 x-r_{1} \\
k-1
\end{array}\right)-\ldots-\left(\begin{array}{c}
2 x-r_{k}-2 \\
2
\end{array}\right)}\right.\right.
\end{aligned}
$$

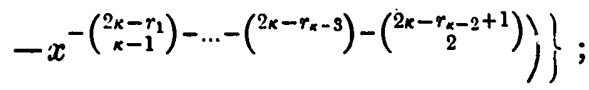

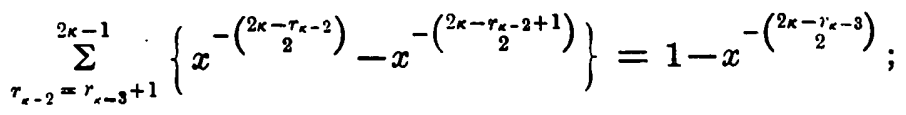

also 
and therefore

$$
\begin{aligned}
& S \equiv \frac{1}{1-x^{-1}}\left\{\sum_{r_{1}, r_{2}, \ldots, r_{k-3}}\left(x^{-\left(\begin{array}{c}
2 \kappa-r_{1} \\
\kappa-1
\end{array}\right)-\ldots-\left(\begin{array}{c}
2 k-r_{x}-3 \\
3
\end{array}\right)}-x^{-\left(\begin{array}{c}
2 k-r_{1} \\
k-1
\end{array}\right)-\ldots-\left(\begin{array}{c}
2 k-r_{k}-3+1 \\
3
\end{array}\right)}\right)\right\}
\end{aligned}
$$

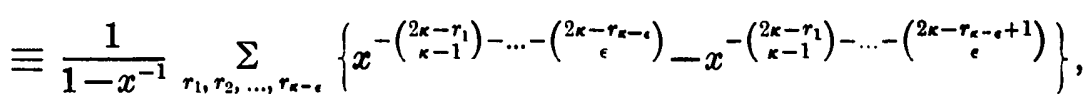

by repeated application of the same method.

Hence, finally,

$$
S \equiv \frac{1}{1-x^{-1}} \sum_{r_{1}=2}^{r_{1}=\kappa+2}\left\{x^{-\left(\begin{array}{c}
2 \kappa-r_{1} \\
\kappa-1
\end{array}\right)}-x^{-\left(\begin{array}{c}
2 \kappa-r_{1}+1 \\
\kappa-1
\end{array}\right)}\right\}=\frac{1}{1-x^{-1}}\left\{1-x^{-\left(\begin{array}{c}
2 \kappa-1 \\
\kappa-1
\end{array}\right)}\right\} \text {. }
$$

So the generating function for $C_{\kappa}^{2}$ is

$$
\begin{gathered}
\frac{x^{2^{2 \kappa-1}-2}}{(1-x)^{2 \kappa-2}} \frac{1-x^{-\left(\begin{array}{c}
2 \kappa-1 \\
\kappa-1
\end{array}\right)}}{1-x^{-1}}=\frac{x^{2^{2 \kappa-1}-1-\frac{1}{(}\left(\begin{array}{c}
2 \kappa \\
\kappa
\end{array}\right)}\left\{1-x^{\frac{3}{2}\left(\begin{array}{c}
2 \kappa \\
\kappa
\end{array}\right)}\right.}{(1-x)^{2 \kappa-1}}, \\
\left(\begin{array}{c}
2 \kappa-1 \\
\kappa-1
\end{array}\right)=\left(\begin{array}{c}
2 \kappa-1 \\
\kappa
\end{array}\right)=\frac{1}{2}\left(\begin{array}{c}
2 \kappa \\
\kappa
\end{array}\right) .
\end{gathered}
$$

since

(ii.) Generating Function for $C_{\kappa} C_{m}$, where $\kappa<m<2 \kappa$.

23. In the first place we suppose the letters of $C_{\kappa}$ and $C_{m}$ to be fixed, and we disregard their arrangement; we seek for products $C_{r} C_{s}(r+s=m+\kappa)$, such that $r$ takes all values from 2 to $\kappa-1$, where each of $C_{r} C_{s}$ contains letters from each of $C_{k} C_{m}$; then, as before, for every such product the minimum weight of $C_{\kappa}$, for the irreducibility of the product $C_{\kappa} C_{m}$, is increased by unity.

The letters of $C_{r}$ may be chosen in $\left(\begin{array}{c}\kappa+m \\ r\end{array}\right)$ ways; but we must exclude the cases where these $r$ letters are all included among the letters of $C_{\kappa}$ or among those of $C_{m}$; hence the total number of such products and therefore of reductions is

$$
\sum_{r=2}^{r=\alpha-1}\left\{\left(\begin{array}{c}
\kappa+m \\
r
\end{array}\right)-\left(\begin{array}{c}
\kappa \\
r
\end{array}\right)-\left(\begin{array}{c}
m \\
r
\end{array}\right)\right\}=\sum_{r=1}^{r=\kappa-1}\left\{\left(\begin{array}{c}
m+\kappa \\
r
\end{array}\right)-\left(\begin{array}{c}
m \\
r
\end{array}\right)\right\}-\left(2^{\kappa}-2\right) .
$$

Independently of these reductions the minimum weight of $C_{x}$ must be $2^{\kappa}-2$, for, if $C_{\kappa}$ is a Jacobian, the product $C_{\kappa} C_{m}$ is certainly reducible ; hence the minimum weight of $C_{k}$ for irreducibility is

$$
\sum_{r=1}^{r=\kappa-1}\left\{\left(\begin{array}{c}
m+\kappa \\
r
\end{array}\right)-\left(\begin{array}{c}
m \\
r
\end{array}\right)\right\} \text {. }
$$


Again, if $C_{m}$ is a Jacobian of the form $\left(C_{l}, C_{m-l}\right)$, then

$$
C_{\kappa} C_{m}=\left(C_{l}, C_{\kappa}\right) C_{m-l}-\left(C_{m-l}, C_{\kappa}\right) C_{l},
$$

and this is a reduction if both $l$ and $m-l$ are less than $\kappa(m<2 \kappa)$. So $l$ may have the values $\kappa-1, \kappa-2, \ldots, m-\kappa+1$ (it is to be remarked that the values $\theta, m-\theta$ of $l$ lead to the same result); now the letters of $C_{m}$ can be chosen for $C_{l}$ in $\left(\begin{array}{c}m \\ l\end{array}\right)$ ways, so that the number of ways in which $C_{m}$ can be chosen to give rise to a Jacobian reduction, whatever be the letters of $C_{k}$, is

$\frac{1}{2}\left\{\left(\begin{array}{c}m \\ \kappa-1\end{array}\right)+\left(\begin{array}{c}m \\ k-2\end{array}\right)+\ldots+\left(\begin{array}{c}m \\ m-\kappa+1\end{array}\right)\right\}$

$$
=\frac{1}{2}\left\{2^{m}-2\right\}-\left\{\left(\begin{array}{c}
m \\
\kappa
\end{array}\right)+\left(\begin{array}{c}
m \\
\kappa+1
\end{array}\right)+\ldots+\left(\begin{array}{c}
m \\
m-1
\end{array}\right)\right\} \text {. }
$$

Moreover, by the perpetuant type theorem, the minimum weight of $C_{m}$ is $2^{n-1}-1$, and all these reductions are independent. Hence, without regarding the arrangement of the letters, we see that the minimum weight of the product $C_{k} C_{m}$ for irreducibility is

$$
\begin{aligned}
\left.\sum_{r=1}^{r=\kappa-1} i\left(\begin{array}{c}
m+\kappa \\
r
\end{array}\right)-\left(\begin{array}{c}
m \\
r
\end{array}\right)\right\}+2^{m}-2 & -\sum_{r=\kappa}^{r=m-1}\left(\begin{array}{c}
m \\
r
\end{array}\right) \\
& =\sum_{r=1}^{r=\kappa-1}\left(\begin{array}{c}
m+\kappa \\
r
\end{array}\right)+2^{m}-2-\left(2^{m}-2\right) \\
& =\sum_{r=1}^{r=\kappa-1}\left(\begin{array}{c}
m+\kappa \\
r
\end{array}\right) .
\end{aligned}
$$

In considering the arrangement of the letters, there is one reduction corresponding to each product $C_{k}^{\prime} C_{m}^{\prime}$ which follows in the sequence of products the product $C_{\kappa} C_{m}$ which we are considering : this is obvious if $C_{\kappa}^{\prime}$ contain letters from $C_{\kappa}$ and $C_{m}$. If, however, $C_{\kappa}^{\prime}$ contain only letters from $C_{m}$, there is still one reduction; for, if the product $C_{k} C_{m}$ is irreducible, $C_{m}$ must not be expressible in terms of a Jacobian of which $C_{x}^{\prime}$ is one of the forms.

Now the total number of forms $C_{\kappa} C_{m}$ is $\left(\begin{array}{c}m+\kappa \\ \kappa\end{array}\right)$, and the extra reductions due to the arrangements of the letters number in the various cases

$$
0,1,2, \ldots,\left(\begin{array}{c}
m+\kappa \\
\kappa
\end{array}\right)-1, \text { respectively ; }
$$


hence the generating function for $C_{k} C_{m}$ is

$$
\begin{aligned}
& \frac{x^{\left(\begin{array}{c}
m+\kappa \\
\kappa-1
\end{array}\right)+\left(\begin{array}{c}
m+\kappa \\
k-2
\end{array}\right)+\ldots+\left(\begin{array}{c}
m+\kappa \\
1
\end{array}\right)\left\{1+x+x^{2}+\ldots+x^{\left(\begin{array}{c}
m+\kappa \\
\kappa
\end{array}\right)-1}\right\}}}{(1-x)^{m+\kappa-2}} \\
& =\frac{x^{\left(\begin{array}{c}
m+\kappa \\
\kappa-1
\end{array}\right)+\left(\begin{array}{c}
m+\kappa \\
k-2
\end{array}\right)+\ldots+\left(\begin{array}{c}
m+\kappa \\
1
\end{array}\right)}\left\{1-x{ }^{\left.\left(\begin{array}{c}
m+\kappa \\
\kappa
\end{array}\right)\right\}}\right.}{(1-x)^{m+\kappa-1}} .
\end{aligned}
$$

This result proves that the generating function for products of degree $\delta$ having no factor of degree less than $(\kappa+1)$, provided $\kappa \geqslant \frac{1}{3} \delta$ (and of course $\leqslant \frac{1}{2} \delta$ ), is

$$
\frac{\left.x^{(\delta} \begin{array}{l}
\delta \\
1
\end{array}\right)+\left(\begin{array}{l}
\delta \\
2
\end{array}\right)+\ldots+\left(\begin{array}{l}
\delta \\
k
\end{array}\right)}{(1-x)^{\delta-1}}
$$

(see also Section V., § 13).

The case of $\kappa=\frac{1}{2} \delta$ requires a word of explanation: the irreducible forms considered in this case are the perpetuant types of degree $\delta$; their generating function is known to be

$$
\frac{x^{2 s-1}-1}{(1-x)^{\delta-1}}
$$

and this has been proved to be exact ; ${ }^{*}$ when $\delta$ is odd and $=2 n+1$, the generating function as given by the preceding result is

$$
\frac{\left.x^{\left({ }^{2 n+1} 1\right.}\right)+\ldots+\left(\begin{array}{c}
2 n+1 \\
n-1
\end{array}\right)+\left(\begin{array}{c}
2 n+1 \\
n
\end{array}\right)}{(1-x)^{2 n}}=\frac{x^{2^{s-1}-1}}{(1-x)^{\delta-1}} \text {. }
$$

A slight exception occurs in the case where $\delta$ is even and $=2 n$; here the generating function is

$$
\frac{x^{2^{d-1}-1}}{(1-x)^{\delta-1}}=\frac{x^{\left(\begin{array}{c}
2 n \\
1
\end{array}\right)+\left(\begin{array}{c}
2 n \\
2
\end{array}\right)+\ldots+\left(\begin{array}{c}
2 n \\
n-1
\end{array}\right)+\frac{1}{(2 n}\left(\begin{array}{c}
2 n \\
n
\end{array}\right)}}{(1-x)^{2 n-1}}
$$

(iii.) Generating Function for $C_{\kappa} C_{2 \kappa}$.

24. Similar methods are applied in the investigation of products $C_{\kappa} C_{2 \kappa}$, but the resulting generating function does not in general admit of ex-

-Wood, "On the Irreducibility of Perpetuant Types," Proc. London Math. Soc., Ser. 2, Vol. 2. SER. 2. VOL. 2. No. 867 : 
pression in a simple form; in the special case of products $C_{8} C_{6}$ we shall evaluate the generating function for the purpose of the subsequent discussion of syzygies of degree 9 .

The number of reductions possible, where the letters of $C_{\kappa} C_{2 \kappa}$ are fixed, is

$$
\sum_{r=2}^{r=x-1}\left\{\left(\begin{array}{c}
3 \kappa \\
r
\end{array}\right)-\left(\begin{array}{c}
2 \kappa \\
r
\end{array}\right)-\left(\begin{array}{l}
\kappa \\
r
\end{array}\right)\right\}+2^{\alpha}-2+2^{2 x-1}-1
$$

for $C_{\kappa}$ must not be a Jacobian, and the minimum weight of $C_{2 \times}$ is $2^{2 k-1}-1$. Hence the minimum weight is, for a fixed choice of letters,

$$
\begin{aligned}
& \sum_{r=1}^{r=k-1}\left(\begin{array}{c}
3 \kappa \\
r
\end{array}\right)+\frac{1}{2}\left(\begin{array}{c}
2 \kappa \\
\kappa
\end{array}\right)-\frac{1}{2}\left(2^{2 \kappa}-2\right)-\left(2^{\kappa}-2\right)+2^{\kappa}-2+2^{2 \kappa-1}-1 \\
= & \sum_{r=1}^{r=k-1}\left(\begin{array}{c}
3 \kappa \\
r
\end{array}\right)+\frac{1}{2}\left(\begin{array}{c}
2 \kappa \\
k
\end{array}\right) .
\end{aligned}
$$

Now suppose that the letters of $C_{k}$ are $a_{r_{1}}, a_{r_{2}}, \ldots, a_{r_{k}}$, where

$$
1<r_{1}<r_{2} \ldots<r_{k}
$$

then the number of products $C_{k}^{\prime} C_{2 k}^{\prime}$, such that $C_{k}^{\prime}$ contains letters of $C_{k}$ and of $C_{2 \kappa}$, which follow the product $C_{\kappa} C_{2 k}$ in the sequence already determined is easily seen to be

$$
N \equiv\left(\begin{array}{c}
3 \kappa-r_{1} \\
\kappa
\end{array}\right)-\left(\begin{array}{c}
2 \kappa-r_{1}+1 \\
\kappa
\end{array}\right)+\left(\begin{array}{c}
3 \kappa-r_{2} \\
\kappa-1
\end{array}\right)+\ldots+\left(\begin{array}{c}
3 \kappa-r_{\kappa} \\
1
\end{array}\right)
$$

and, as before, we can show that

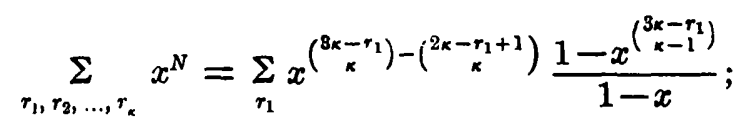

here $r_{1}$ may have any value from 2 to $2 \kappa+1$.

If $C_{k}$ contains $a_{1}$ (corresponding to $r_{1}=1$ ), we get a term

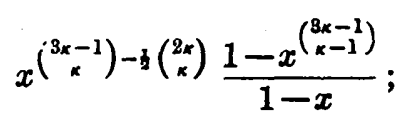

where the extra index $\frac{1}{2}\left(\begin{array}{c}2 \kappa \\ \kappa\end{array}\right)$ arises from the fact that, if $C_{2 \kappa}$ does not contain $a_{1}$ and can be expressed as a Jacobian $\left(C_{k}, C_{k}^{\prime}\right)$, there is a reduction, 
Hence the generating function for irreducible products $C_{\kappa} C_{2 \kappa}$ is

$$
\begin{aligned}
& \frac{x^{\left(\begin{array}{c}
3 \kappa \\
1
\end{array}\right)+\left(\begin{array}{c}
3 \kappa \\
2
\end{array}\right)+\ldots+\left(\begin{array}{c}
3 \kappa \\
\kappa-1
\end{array}\right)+1\left(\begin{array}{c}
2 \kappa \\
\kappa
\end{array}\right)}}{(1-x)^{3 k-1}} \cdot x^{\left(\begin{array}{c}
3 \kappa-1 \\
\kappa
\end{array}\right)-\frac{1}{2}\left(\begin{array}{c}
2 \kappa \\
\kappa
\end{array}\right)}\left(1-x^{\left(\begin{array}{c}
3 \kappa-1 \\
\kappa-1
\end{array}\right)}\right) \\
& +\sum_{r=2}^{r=2 \kappa+1} x^{\left(\begin{array}{c}
3 \kappa-r \\
\kappa
\end{array}\right)-\left(\begin{array}{c}
2 k-r+1 \\
\kappa
\end{array}\right)}\left(1-x^{\left(\begin{array}{c}
3 k-r \\
\kappa-1
\end{array}\right)}\right) ! \text {. }
\end{aligned}
$$

Putting $\kappa=3$, we find for the irreducible products $C_{3} C_{6}$ of degree 9 the generating function

$$
\frac{x^{55}\left(1-x^{10}+x^{9}-x^{19}+x^{16}-x^{31}+x^{25}-x^{74}\right)}{(1-x)^{x}} .
$$

Summary of Results giving Generating Functions for the Enumeration of certain Classes of Irreducible Products of total Degree $\delta$.

Products.

All products containing no factor $C,(\oint 14)$

(where $\delta=2 n)(\oint 15)$

$$
C_{2}^{m} r_{3}
$$

(where $\delta=2 m+3)(\oint 18)$

$C_{2}^{m} C_{4}$

$($ where $\delta=2 m+4)(\oint 19)$

$C_{3}^{m} P_{d-2 m}\left(P_{b-2 m}\right.$ contains no factor of degree leas than 3 and is neither $C_{3}$ nor $\left.C_{4}\right)(\$ 17)$

All products containing no factor of degree less than $3(\$ 20)$

$$
C_{*}^{2}
$$

(where $\delta=2 \kappa)(\$ 22)$

$$
\begin{gathered}
C_{k} C_{m} \\
(\text { where } \delta=\kappa+m, \\
\kappa<m<2 \kappa)(\oint 23)
\end{gathered}
$$

Generating Functions.

$$
\begin{aligned}
& (1-x)^{\delta / \overline{1}} \\
& \frac{x^{\delta}\left(1-x^{3}\right)\left(1-x^{5}\right) \ldots\left(1-x^{\delta-1}\right)}{(1-x)^{\delta-1}} \\
& \frac{x^{8}\left(1-x^{3}\right)\left(1-x^{5}\right) \ldots\left(1-x^{2-2}\right)}{(1-x)^{1-1}}\left\{1+\frac{x^{3}\left(1-x^{6-3}\right)}{1-x^{2}}+x^{6} \frac{\left(1-x^{4-3}\right)\left(1-x^{6-1}\right)}{\left(1-x^{2}\right)\left(1-x^{3}\right)}\right\} \\
& \begin{array}{c}
x^{\delta+3}\left(1-x^{3}\right)\left(1-x^{5}\right) \ldots\left(1-x^{\delta-\delta}\right)\left(1-x^{\delta-3}\right)\left(1-x^{\delta-2}\right)\left(1-x^{\delta-1}\right) \\
(1-\bar{x})^{\delta-1}\left(1-x^{-2}\right)\left(1-x^{3}\right)
\end{array} \\
& \times\left\{1+\begin{array}{c}
x^{i}\left(1-x^{4-4}\right) \\
\left(1-x^{-1}\right)
\end{array}\right\}
\end{aligned}
$$

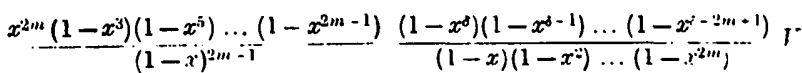

$$
\begin{aligned}
& \text { (where } V \text { is the generating function of } P_{\delta}-3_{m} \text { ) }
\end{aligned}
$$

$$
\begin{aligned}
& x\left(\begin{array}{l}
\left(\begin{array}{l}
0 \\
1
\end{array}\right)+\left(\begin{array}{l}
\dot{b} \\
2
\end{array}\right) \\
(1)=x
\end{array}\right. \\
& (\overline{1-x})^{j-1}
\end{aligned}
$$

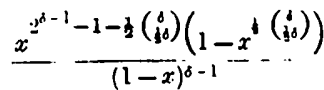

$$
\begin{aligned}
& \frac{x\left(\begin{array}{l}
\delta \\
1
\end{array}\right)+\left(\begin{array}{l}
\delta \\
2
\end{array}\right)+\ldots+\left(\begin{array}{c}
\delta \\
x-1
\end{array}\right)\left(1-x^{(\delta}\left(\begin{array}{l}
\delta \\
\alpha
\end{array}\right)\right)}{(1-x)^{\delta-1}}
\end{aligned}
$$


VII. Syzygies of Degrees 6, 7, and 8.-No'te on Syzyares of Degree 9.

25. We are now in a position to write down from the preceding general results the generating functions for the enumeration of the irreducible products of degrees 6,7 , and 8 respectively.

\section{Degree 6.}

Products. Generating Functions.

How obtained.

\begin{tabular}{|c|c|c|c|}
\hline Trpe forms :- & $C_{6}$ & $\frac{x^{31}}{(1-x)^{5}}$ & Perpetuant type theorem \\
\hline \multirow[t]{10}{*}{ Products :- } & $C_{3}^{2}$ & $\frac{x^{21}-x^{31}}{(1-x)^{5}}$ & $C_{\kappa}^{2}, \kappa=3$ (Section VI., $\left.\$ 22\right)$ \\
\hline & $C_{2} C_{4}$ & $\frac{x^{9}+x^{11}-x^{14}-x^{21}}{(1-x)^{5}}$ & $C_{2}^{n_{2}} C_{4}, m=1$ (Section V., $\left.\$ 19\right)$ \\
\hline & $C_{2}^{3}$ & $\frac{x^{6}-x^{9}-x^{11}+x^{14}}{(1-x)^{5}}$ & $C_{2}^{m}, m=3$ (Section V., $\left.\$ 15\right)$ \\
\hline & $C_{1} C_{5}$ & $\frac{6 x^{15}}{(1-x)^{4}}$ & Perpetuant type theorem \\
\hline & $C_{1} C_{2} C_{3}$ & $\frac{6\left(x^{5}-x^{15}\right)}{(1-x)^{4}}$ & $C_{2} C_{8}, m=1$ (Section V., $\left.\S 18\right)$ \\
\hline & $C_{1}^{2} C_{4}$ & $\frac{15 x^{7}}{(1-x)^{3}}$ & Perpetuant type theorem \\
\hline & $C_{1}^{2} C_{2}^{2}$ & $\frac{15\left(x^{4}-x^{7}\right)}{(1-x)^{9}}$ & $C_{2}^{m}, m=2$ (Section V., $\left.\$ 15\right)$ \\
\hline & $C_{1}^{3} C_{3}$ & $\frac{20 x^{3}}{(1-x)^{2}}$ & Perpetuant type theorem \\
\hline & $C_{1}^{4} C_{2}$ & $\frac{15 x}{1-x}-10 x$ & $C_{1}^{n-2} C_{2}, n=6$ (Section V., $\left.\S 14\right)$ \\
\hline & $C_{1}^{6}$ & 1 & \\
\hline
\end{tabular}

Also-

(i.) Sum of generating functions for forms $C_{6}, C_{3}^{2}$ is $x^{21} /(1-x)^{5}$ (see Section V., \$13).

(ii.) Sum of generating functions for forms $C_{6}, C_{3}^{2}, C_{2} C_{4}, C_{2}^{3}$ is $x_{i}^{6} /(1-x)^{5}$ (see Section V., $§ 13$ ). 
(iii.) Sum of all generating functions is $1 /(1-x)^{5}$, so that all the enumeration of the irreducible forms is exact and all the syzygies for degree 6 have been identified.

One point calls for comment: in reducing the products $C_{2} C_{4}$, if $C_{2}=\left(a_{5} a_{6}\right)^{\nu}$, the only restriction on $\nu$ is (Section V., $\left.\S 19\right) \nu \geqslant 2$. Now consider the form

$$
C_{2} C_{4}=\left(a_{5} a_{6}\right)^{2}\left\{\left(a_{1} a_{2}\right)^{3},\left(a_{3} a_{4}\right)^{3}\right\},
$$

which arises in products of weight 9 . By the ordinary Jacobian identity we have

$\left(a_{5} a_{6}\right)^{2}\left\{\left(a_{1} a_{2}\right)^{3},\left(a_{9} a_{4}\right)^{8}\right\} \equiv\left(a_{1} a_{2}\right)^{8}\left\{\left(a_{5} a_{6}\right)^{2},\left(a_{3} a_{4}\right)^{3}\right\}-\left(a_{3} a_{4}\right)^{3}\left\{\left(a_{5} a_{6}\right)^{2},\left(a_{1} a_{2}\right)^{3}\right\}$.

Now each of the Jacobians $\left\{\left(a_{5} a_{6}\right)^{2},\left(a_{3} a_{4}\right)^{9}\right\},\left\{\left(a_{5} a_{6}\right)^{2},\left(a_{1} a_{2}\right)^{3}\right\}$ is of degree 4 and weight 6, and therefore is expressible as a sum of product forms. Hence the product $\left(a_{5} a_{6}\right)^{2}\left\{\left(a_{1} a_{2}\right)^{3},\left(a_{8}^{\prime} a_{4}\right)^{3}\right\}$ is reducible: this is not in opposition to the general result, as we can shew that the Jacobian $\left\{\left(a_{1} a_{2}\right)^{3},\left(a_{3} a_{4}\right)^{3}\right\}$, although of degree 4 and weight 7 , is itself reducible. For, by the perpetuant type theorem,

$$
\begin{aligned}
& \left\{\left(a_{1} a_{2}\right)^{8},\left(a_{3} a_{4}\right)^{8}\right\} \\
& \equiv \\
& \equiv\left(a_{1} a_{2}\right)^{4}\left(a_{2} a_{9}\right)^{2}\left(a_{9} a_{4}\right)+\text { product forms } \\
& \\
& \quad \kappa\left\{\left(a_{1} a_{2}\right)^{4},\left(a_{3} a_{4}\right)\right\}^{2}+\lambda\left\{\left(a_{1} a_{2}\right)^{5},\left(a_{3} a_{4}\right)\right\}+\mu\left\{\left(a_{1} a_{2}\right)^{4},\left(a_{3} a_{4}\right)^{2}\right\} \\
& \quad+\text { product forms, }
\end{aligned}
$$

and every term of $\left\{\left(a_{1} a_{2}\right)^{5},\left(a_{3} a_{4}\right)\right\}$ is a product form. Hence $\left\{\left(a_{1} a_{2}\right)^{3},\left(a_{8} a_{4}\right)^{9}\right\} \equiv \kappa\left\{\left(a_{1} a_{2}\right)^{4},\left(a_{3} a_{4}\right)\right\}^{2}+\mu\left\{\left(a_{1} a_{2}\right)^{4},\left(a_{3} a_{4}\right)^{2}\right\}$ +product forms. In this result interchange $a_{1}$ and $a_{2}$ and subtract ; then

$$
2\left\{\left(a_{1} a_{2}\right)^{3},\left(a_{3} a_{4}\right)^{3}\right\}=\text { product forms ; }
$$

and therefore the Jacobian is reducible.

In the treatment of product forms of degree $3 \kappa(\kappa \geqslant 3)$ no such difficulty can arise, for, although we have the analogous result

$$
C_{\kappa}^{\prime}\left(C_{\kappa}, \bar{C}_{\kappa}\right)=\Sigma \text { (product of three factors), }
$$

where $C_{\kappa}^{\prime}$ is of weight $2^{2 \kappa-2}-2$, and each of $C_{k}, \bar{C}_{\kappa}$ is of weight $2^{2 \kappa-2}-1$, it will be seen that the minimum weight, as determined in Section V., $\$ 24$, of the factor $C_{\kappa}^{\prime} C_{2 \kappa}$ is in all cases greater than $2^{2 \kappa-2}-2$; so that there is no occasion to investigate the reducibility of the Jacobian form $\left(C_{\kappa}, \breve{C}_{k}\right)$ of weight $2^{2 \kappa-1}-1$ and degree $2 \kappa$ (see $\$ 24$ ). 


\section{Degree 7.}

26.

Products. Generating Functions.

Type forms:- $\quad C_{\eta}$

$$
\frac{x^{69}}{(1-x)^{6}}
$$

Products :- $\quad C_{3} C_{4}$

$$
\begin{array}{lc}
C_{2} C_{5} \quad \frac{x^{17}\left(1-x^{7}\right)\left(1+x^{2}+x^{4}\right)}{(1-x)^{6}} \\
C_{2}^{2} C_{3} \frac{x^{7}\left(1-x^{5}\right)\left(1-x^{7}\right)\left(1+x^{5}+x^{7}\right)}{(1-x)^{6}}
\end{array}
$$$$
C_{1} C_{6}
$$$$
\frac{7 x^{31}}{(1-x)^{5}}
$$

$C_{1} C_{3}^{2}$

$$
\frac{7\left(x^{21}-x^{31}\right)}{(1-x)^{5}}
$$$$
C_{1} C_{2} C_{4} \quad \frac{7\left(x^{9}+x^{11}-x^{14}-x^{21}\right)}{(1-x)^{5}}
$$$$
C_{1} C_{2}^{3} \quad \frac{7\left(x^{6}-x^{9}-x^{11}+x^{14}\right)}{(1-x)^{5}}
$$

$$
C_{1}^{2} C_{5}
$$$$
C_{1}^{2} C_{2} C_{3}
$$$$
\frac{21 x^{15}}{(1-x)^{4}}
$$

How obtained.

Perpetuant type theorem

$C_{\kappa} C_{m}, \kappa=3, m=4$

(Section VI., § 23)

$C_{2}^{m} C_{r}, m=1, r=5$

(Section V., § 17)

$C_{2}^{m} C_{3}, \quad m=2$

(Section V., § 18)

$$
\frac{21\left(x^{5}-x^{15}\right)}{(1-x)^{4}}
$$

Results for degree 6

$C_{1}^{3} C_{4}$

$C_{1}^{3} C_{2}^{2}$

$$
\frac{35 x^{7}}{(1-x)^{8}}
$$

$C_{1}^{3} C_{2}^{2}$
$C_{1}^{4} C_{3}$

$$
\frac{35\left(x^{4}-x^{7}\right)}{(1-x)^{8}}
$$

$C_{1}^{5} C_{2}$

$$
\frac{35 x^{8}}{(1-x)^{2}}
$$

$C_{1}^{7}$

$$
\frac{21 x}{1-x}-15 x
$$

$C_{1}^{n-2} C_{2}, \quad n=7$

(Section V., § 14)

Also-

(i.) Sum of generating functions for forms $C_{7}, C_{9} C_{4}$ is $x^{28} /(1-x)^{6}$ (see Section V., § 13). 
(ii.) Sum of generating functions for forms $C_{7}, C_{3} C_{4}, C_{2} C_{5}, C_{2}^{2} C_{3}$ is $x^{7} /(1-x)^{6}$ (see Section V., $\S 13$ ).

(iii.) Sum of all generating functions is $1 /(1-x)^{6}$; so that all the syzygies are enumerated above.

\section{Degree 8.}

It is clear that we need only determine the generating functions for the products $C_{8}, C_{4}^{2}, C_{3} C_{5}, C_{2} C_{6}, C_{2} C_{3}^{2}, C_{2}^{2} C_{4}, C_{2}^{4}$; since all other products have a factor $C_{1}$, and the corresponding generating functions may be determined from the results for degree 7 (see Section V., § 14).

Products.

Generating Functions.

Type forms:- $\quad C_{8}$

Products :- $\quad C_{4}^{2} \quad \frac{x^{92}-x^{127}}{(1-x)^{7}}$

$$
\begin{array}{cc}
C_{3} C_{5} & \frac{x^{36}-x^{92}}{(1-x)^{7}} \\
C_{2} C_{6} & \frac{x^{2}\left(1-x^{7}\right)\left(1-x^{8}\right)}{\left(1-x^{2}\right)(1-x)^{2}} \frac{x^{31}}{(1-x)^{5}} \\
C_{2} C_{3}^{2} & \frac{x^{2}\left(1-x^{7}\right)\left(1-x^{8}\right)}{\left(1-x^{2}\right)(1-x)^{2}} \frac{x^{21}-x^{31}}{(1-x)^{5}} \\
C_{2}^{2} C_{4} & \frac{x^{11}\left(1-x^{5}\right)\left(1-x^{6}\right)\left(1-x^{14}\right)}{\left(1-x^{2}\right)(1-x)^{7}} \\
C_{2}^{4} & \frac{x^{8}\left(1-x^{3}\right)\left(1-x^{5}\right)\left(1-x^{7}\right)}{(1-x)^{7}}
\end{array}
$$

How obtained.

Perpetuant type theorem $C_{\kappa}^{2}, \kappa=4$ (Section VI., $\$ 22$ ) $C_{\kappa} C_{n,}, \kappa=3, m=5$ (Section VI., § 23) $C_{2}^{n n} C_{r}, m=1, r=6$

(Section V., § 17) $C_{2}^{m} C_{r}, m=1, r=6$ (Section V., \$ 17)

$$
C_{2}^{m} C_{4}, m=2
$$

(Section V., § 19)

$$
C_{2}^{m}, m=4
$$

(Section V., § 15)

Also-

(i.) Sum of generating functions for forms $C_{8}, C_{4}^{2}$ (containing no factor of degree 3 or less) is

$$
\frac{x^{92}}{(1-x)^{7}}=\frac{x^{\left(\begin{array}{l}
8 \\
1
\end{array}\right)+\left(\begin{array}{l}
8 \\
2
\end{array}\right)+\left(\begin{array}{l}
8 \\
3
\end{array}\right)}}{(1-x)^{7}}
$$

(ii.) Sum of generating functions for forms $C_{8}, C_{4}^{2}, C_{3} C_{5}$ is $x^{86} /(1-x)^{7}$ (see Section V., $\$ 13$ ).

(iii.) Sum of all generating functions above is $x^{8} /(1-x)^{7}$ (see Section V., § 13). 


\section{Note on Syzygies of Degree 9.}

27. The only types and products of degree 9 which have no factor of degree less than 3 are $C_{9}, C_{4} C_{5}, C_{3} C_{6}, C_{3}^{3}$; and therefore the generating function for all such irreducible forms is $x^{45} /(1-x)^{8}$. Also the generating function for $C_{9}$ is $x^{255} /(1-x)^{8}$ : the generating function for $C_{4} C_{5}$ is $\frac{x^{129}\left(1-x^{126}\right)}{(1-x)^{8}}$, while the generating function for $C_{3} C_{6}$ is $(\S 24)$

$$
\frac{x^{65}\left(1-x^{10}+x^{9}-x^{19}+x^{16}-x^{31}+x^{25}-x^{74}\right)}{(1-x)^{8}} .
$$

Hence the generating function for the products $C_{3}^{3}$ should be

$$
\begin{aligned}
& \frac{x^{45}-x^{255}-x^{129}\left(1-x^{126}\right)-x^{55}\left(1-x^{10}+x^{9}-x^{19}+x^{16}-x^{91}+x^{25}-x^{74}\right)}{(1-x)^{8}} \\
& =\frac{x^{45}\left(1-x^{10}+x^{20}-x^{19}+x^{29}-x^{26}+x^{41}-x^{35}\right)}{(1-x)^{8}} .
\end{aligned}
$$

This generating function may be written in the form

$$
\begin{aligned}
& x^{45}\left(1+2 x+3 x^{2}+4 x^{3}+5 x^{4}+6 x^{5}+7 x^{6}+8 x^{7}+9 x^{8}+10 x^{9}+10 x^{10}+10 x^{11}\right. \\
& +10 x^{12}+10 x^{18}+10 x^{14}+10 x^{15}+10 x^{16}+10 x^{17}+10 x^{18}+9 x^{19}+9 x^{20} \\
& +9 x^{21}+9 x^{22}+9 x^{29}+9 x^{24}+9 x^{25}+8 x^{26}+7 x^{27}+6 x^{28}+6 x^{29}+6 x^{30}+6 x^{91} \\
& \left.+6 x^{82}+6 x^{93}+6 x^{34}+5 x^{85}+4 x^{36}+3 x^{37}+2 x^{38}+x^{39}\right) \\
& (1-x)^{6}
\end{aligned}
$$

The sum of the coefficients in the numerator is 280 , the same as the number of possible different arrangements of the letters in the factors of $C_{3}^{3}$.

The first fow terms may be accounted for by the methods already used, thus

$$
\begin{aligned}
& \left(a_{1} a_{2} a_{9}\right)\left(a_{4} a_{5} a_{6}\right)\left(a_{7} a_{8} a_{9}\right) \text { is of minimum weight } 45 \text {, } \\
& \left.\left(a_{1} a_{2} a_{4}\right)\left(a_{3} a_{5} a_{6}\right)\left(a_{7} a_{8} a_{9}\right)\right\} \\
& \left(a_{1} a_{2} a_{5}\right)\left(a_{9} a_{4} a_{6}\right)\left(a_{7} a_{8} a_{9}\right) \\
& \left(a_{1} a_{2} a_{4}\right)\left(a_{8} a_{5} a_{7}\right)\left(a_{6} a_{8} a_{9}\right) \\
& \left(a_{1} a_{2} a_{8}\right)\left(a_{4} a_{5} a_{8}\right)\left(a_{6} a_{7} a_{9}\right) \\
& \begin{array}{l}
\left(a_{1} a_{2} a_{6}\right)\left(a_{8} a_{4} a_{5}\right)\left(a_{7} a_{8} a_{9}\right) \\
\left(a_{1} a_{2} a_{5}\right)\left(a_{9} a_{4} a_{7}\right)\left(a_{6} a_{8} a_{9}\right)
\end{array} \\
& \left(a_{1} a_{2} a_{4}\right)\left(a_{8} a_{5} a_{8}\right)\left(a_{6} a_{7} a_{9}\right) \\
& \left(a_{1} a_{2} a_{8}\right)\left(a_{4} a_{5} a_{9}\right)\left(a_{6} a_{7} a_{8}\right)
\end{aligned}
$$


But our known reductions give six products of minimum weight 49 (instead of five products) : they are

$$
\begin{aligned}
& \left(a_{1} a_{2} a_{7}\right)\left(a_{3} a_{4} a_{5}\right)\left(a_{6} a_{8} a_{9}\right), \\
& \left(a_{1} a_{2} a_{6}\right)\left(a_{9} a_{4} a_{7}\right)\left(a_{5} a_{8} a_{9}\right), \\
& \left(a_{1} a_{2} a_{5}\right)\left(a_{3} a_{4} a_{8}\right)\left(a_{6} a_{7} a_{9}\right), \\
& \left(a_{1} a_{2} a_{4}\right)\left(a_{9} a_{5} a_{9}\right)\left(a_{6} a_{7} a_{8}\right), \\
& \left(a_{1} a_{2} a_{9}\right)\left(a_{4} a_{6} a_{7}\right)\left(a_{5} a_{8} a_{9}\right), \\
& \left(a_{1} a_{8} a_{4}\right)\left(a_{2} a_{5} a_{6}\right)\left(a_{7} a_{8} a_{9}\right),
\end{aligned}
$$

and one of these forms should be of minimum weight 50 at least.

This would seem to imply that Stroh's syzygies of degree 4 as used hitherto are insufficient for the investigation of the products $C_{3}^{3}$ : it seems probable that similar difficulties will arise successively in the treatment of products $C_{4}^{4}, C_{5}^{5}, \ldots, C_{m}^{m}, \ldots$, that is, whenever we arrive at a degree $m^{2}$.

[Note.-The symbol $\left(a_{1} a_{2} a_{9}\right)$ is used simply to denote any covariant linear in the coefficients of each of the quantics represented by the letters $\left.a_{1}, a_{2}, a_{8} \cdot\right]$ 